\title{
Les leçons de la Réunion préparatoire de Porto Alegre pour la Conférence Spécialisée de Droit International Privé - CIDIP VII - SUR LA PROTECTION DES CONSOMMATEURS ET DES NÉGOCIATIONS SUBSÉQUENTES ${ }^{* 1}$
}

\section{Cláudia Lima Marques ${ }^{2}$}

\section{Introduction}

En décembre 2006 eût lieu, à l'Université Fédérale du Rio Grande do Sul (UFRGS -Porto Alegre, Brésil), la Réunion Préparatoire de la Conférence Spécialisée en Droit International Privé (CIDIP VII) sur la protection des consommateurs sous les auspices de l'OÉA, du Ministère de la Justice et du Ministère des Relations Étrangères du Brésil, grâce à la collaboration du gouvernement de l'État du Rio Grande do Sul et sous l'organisation de la UFRGS et de l'Instituto Brasileiro de Política e Direito do Consumidor-Brasilcon (Brasília) ${ }^{3}$.

* Traduit du portugais par Hughes Brisson, étudiant à l'Université du Québec à Montreal (UQAM) au bac. en relations intemationales et droit intemational, durant son année de stage et d'échange à l'Université Fédérale du Rio Grande do Sul (UFRGS).

1 Étude préparée pour les «Jornadas de Protección al Consumidon, du CEDEP (Centro de Estudios de Derecho, Economiay Politica), Paraguay, octobre 2007, CEDEP, Assunción, Paraguay. Publié en portugais, "As lições da reunião preparatória de Porto Alegre da Conferência Especializada de Direito Intemacional Privado-CIDIP VII de proteção dos consumidores e das negociações posteriores ", in FERNANDEZ ARROYO, Diego and MORENO RODRIGUEZ, José A. Proteción de los Consumidones en America-Trabajos de la CIDIP VII (OEA), Le Ley-CEDEP. Asunción, 2007, p. 179-208. Traduction de Hughes Brisson (UQUÀMMontrèal, UFRGS-Porto Alegre). Les opinions exprimées dans cette étude ne sont pas officielles et ne représentent pas nécéssaiment celles de la délégation brésilienne pour la CIDIP VII. Elles sont mes opinions personnelles en tant que Professeure titulaire de Droit International Privé à l'Université Fédérale du Rio Grande do Sul (UFRGS) et ne découlent pas d'appuis, de pressions ou de consultations de la part du gouvernement brésilien.

2 Professeure titulaire de Droit International Privé à l'Université Fédérale du Rio Grande do Sul, Docteure en Droit (Université de Heidelberg, Allemagne). Maitrise en Droit Civil et Droit International Privé (Université de Tubingen, Allemagne) et spécialisée en Intégration Européenne (Europa-Institut, Saarbrucken, Allemagne). Ex-présidente de l'Instituto Brasileiro de Politica e Direito do Consumidor. Directrice de l'Association internationale de Droit de la Consommation, Bruxelles. Directrice de la International Lau. Association - Brazilian Branch, Londres. Directrice de la DLJV Associaģão Luso-Germânica de Juristas, Berlin. Déléguée brésilienne lors de la réunion préparatoire pour la CIDIP VII sur la protection des consommateurs.

3 La convocation se lisait comme suit (traduction libre) : «La Présidence de la Commissions des questions juridiques et politiques a l'honneur de s'adresser aux Missions permanentes et aux Missions observatoires permanentes de L'OÉA en rapport aux travaux préparatoires pour la septième Conférence spécialisée interaméricaine sur le droit intemacional privé (CIDIP-VII) et se permet de les informer que, dans le but de continuer les travaux préparatoires de la CIDIP-VII dont la méthodologie fut établie par le Conseil Permanent de l'OÉA (document CP/CAJP-2309-05), le Département de Protection et de défense du consommateur du Secrétariat de droit économique du Ministère de la Justice du Brésil (DPDC/SDE/MJ), avec la collaboration de lUniversité Fédérale du Rio Grande do Sul (UFRGS) et l'Institut brésilien de politique et de droit du consommateur (BRASILCON), organisent une réunion préparatoire. Lors de cette réunion seront discutés les documents aprouvés suivants: 1) la proposition des États-Unis d'Amérique (ÉUA) d'une «loi modèle sur les mécanismes de restitution monétaire pour les consommateurs»; 2) la proposition du Brésil intitulée «Une convention interaméricaine sur la loi aplicable à certains contrats et relations de consommation» et 3) la proposition du Canada d'une «loi-modèle sur la juridiction et les règles de conflit de lois dans les contrats de consommation». La réunion se tiendra à l'UFRGS dans la ville de Porto Alegre au Brésil, les 2, 3 et 4 décembre 2006» 
Le but de cet article est d'apporter une réflexion sur les leçons qui peuvent être tirées de cette importante réunion préparatoire, laquelle permit une analyse des trois propositions pour la conférence finale de la CIDIP-VII sur la protection des consommateurs (les proposition brésilienne, canadienne et étasunienne), ainsi que sur les négociations qui suivirent au sein de l'informel «groupe de travail de rédaction» ${ }^{4}$.

Ainsi, j'aimerais diviser cette étude en deux parties : la première, plus générale, sur la réunion de Porto Alegre, ses antécédents et les avancements qu'elle engendra. La seconde partie cherche à dégager les consensus généraux (ou les leçons) atteints autour des propositions présentées par le Brésil, le Canada et les États-Unis d'Amérique (ÉUA) lors de la réunion, les dialogues possibles ainsi que l'état actuel de la question.

Avant de commencer ce rapport, $j$ 'aimerais d'abord vous offrir mon témoignage personnel sur l'énorme évolution de la proposition brésilienne, d'autant plus que je fus l'auteure de la proposition initiale en $2000^{5}$. Cette proposition d'une «Convention Interaméricaine sur le droit applicable à certains contrats et relations de consommation», approuvée par le gouvernement brésilien, se retrouve aujourd'hui grandement améliorée grâce aux contributions, aux suggestions et aux critiques des experts et des délégués présents au Forum Virtuel, à la réunion de Porto Alegre et lors des négociations qui suivirent. Cet article, d'une certaine façon, est un remerciement aux collègues, aux experts et aux délégués m'ayant accompagnée au long de ce cheminement.

Je voudrais remercier particulièrement, non seulement en tant que membre de la délégation brésilienne mais aussi en tant qu'académiste, toutes ces excellentes et brillantes suggestions et ce dialogue empreint de tolérance et de sincérité, qui a permis de construire un échange d'opinions riche, ouvert et fort fructueux. Celui-ci permit de démontrer la profonde bonne volonté et le grand intérêt porté envers la protection des consommateurs de la part de toutes les honorables délégations et des experts indépendants (d'Argentine, d'Uruguay, du Paraguay, d'El Salvador, de la Bolivie, du Pérou, du Costa Rica, de Panama, du Mexique, des États-Unis et du Canada) qui participèrent à ce processus. Je profite aussi de l'occasion pour remercier mes collègues brésiliens. Je suis consciente que la proposition brésilienne (qui en est maintenant à sa

4 Voir WILSON, John. «Introducción al Sistema Interamericano de Derecho Internacional Privado: El proceso de la Séptima Conferencia Especializada Interamericana sobre Derecho Internacional Privado (CIDIP VII) y la cooperación internacional em matéria de Derecho Internacional Privadon, in Cadernos do Programa de Pós-Graduaşão em Dineito-PPGDin. UFRGS, N.V, mars 2006, p.13-19 ouwww oas.org.

5 Voirla proposition originale d'une CIDIP sur la protection des consommateurs in LIMA MARQUES, Claudia. «A insuficiente proteção do consumidor nas normas de Direito Internacional Privado - Da necessidade de uma Convenção Interamericana (CIDIP) sobre a lei aplicável a alguns contratos e relações de consumo", in Retista do Tribunais (São Paulo), vol. 788, juin 2001, année 90, p.11-56. Aussi publiée en espagnol en Argentine : «A insuficiente proteção do consumidor nas normas de DIPr.-Da necessidade de uma Convenção Interamericana sobre a lei aplicável a alguns contratos e relações de consumon, in El Futuro de la codifficación del Derecho internacional privado en America - De la CIDIPVI a la CIDIP VII, Fernández Arroyo, Diego e Mastrangelo, Fabio (org), Alveroni : Córdoba, Argentine, 2005, p.105-165. Et, finalement, publiée au Canada en anglais et en français : "Consumer Protection in Private International Law Rules : the need for an interamerican Convention on the law aplicable to some consumer contracts and consumer transactions (CIDIP)m, in Regards croisés sur les enjeux contemporains du droit de la consommation, Thierry Bourgoignie (dir.), Éditions Yvon Blais, Cowansville (Québec), Canada, 2006, p.145-190. 
quatrième et finale version) se trouve aujourd'hui consolidée et plus mûre grâce à ce dialogue vertueux et fructueux. D'autant plus que, durant mon cours à l'OÉA au mois d'août 2007, les 5 cas hypothétiques impliquant la proposition brésilienne, gentiment envoyés par l'honorable délégation étasunienne, ont pu être résolus par les étudiants et diplomates - de 15 pays membres différents de l'OÉA en moins de 10 minutes, démontrant ainsi la fonctionnalité et l'efficacité que la codification d'une norme semblable au sein des Amériques permettrait. La dette du mouvement de défense des droits des consommateurs envers ce groupe d'intellectuels, d'experts et délégués et envers leur sérieux et fructueux travail est importante. Voyons donc maintenant les détails sur la réunion.

\section{I - La réunion préparatoire de la CIDIP VII sur la protection des consommateurs: antécédents, textes présentés et avancées réalisées.}

Du premier au six décembre 2006, se réunirent à Porto Alegre cinquante experts et délégués de 11 pays des Amériques (Argentine, Bolivie, Brésil, Canada, Costa Rica, El Salvador, États-Unis, Mexiquè, Panama, Paraguay, Uruguay), quelques invités spéciaux provenant de diverses organisations, entre autres de la Conférence de Droit International de LaHaye (Pays-Bas), de Consumers International (Londres), de la International Association of Consumer Law (Belgique/Canada), de la International Association of Judges (Rome) et de la International Law Association (Londres) ${ }^{6}$, ainsi que des représentants de vingt-cinq universités provenant du Brésil, d'Argentine, d'Uruguay, du Paraguay, d'Allemagne (dont l'Institut Max-Planck de Hambourg), d'Italie et d'Amérique du Nord. La réunion fut ouverte par un discours du président mondial de Consumers International (Londres, anciennement IUCO) qui réunit plus de 200 associations de défense des consommateurs de 105 pays. Ensuite, fut lu un message du président de la International Association of Consumer Law (Belgique/ Canada), une organisation non gouvernementale à vocation scientifique, souhaitant succès à la réunion et aux travaux préparatoires de la CIDIP-VII sur la thématique de la protection des consommateurs.

Nous nous pencherons maintenant sur les détails de cette réunion, ses antécédants, les textes qui y furent présentés ainsi que leur version finale (jusqu'à maintenant du moins) et essaierons d'identifier quel fut' le consensus atteint ou les leçons qui peuvent en être retenues.

\footnotetext{
6 Étaient aussi présents, en tant qu’observateurs, des représentants du Mercosur et d'organismes brésiliens intéréssés par la défense intemationale des consommateurs tels que : Sociedade Brasileira de Direito Internacional, IDEC-Instituto de Defesa do Consumidor (São Paulo), Forum Brasileiro das Associaşões de Defesa do Consumidor (São Paulo), Conselho Federal da Ordem dos Adrogados do Brasil (Brasilia), Crdem dos Advogados do Brastl-Subsegão Rio Grande do Sul, Forum Rio Grandense das Associagẽes de Defesa do Consumidor (Porto Alegre), Associação das Donas de Casa e Consumidones do RS, Camara de Comérico Americana-American Chamber of Comence (Porto Alegre), ACHANC, Assaciagão Brasiliera de Direito da Informática-ABDI, (São Paulo), Associaģão Brasileira de Coméncio eletrónicoABCE, Tribunal de Justiga do RS, TRF4 Regiäo, Ministério Público do Estado do Rio Grande do Sul, Defensoria Pública do RS, Procon-RS et plusieurs autres. Le Secrétariat de la ALADI ne put être présent puisqu'il y avait, au même moment, une réunion à Montevideo. UNIDROIT préféra ne pas envoyer de représentant et l'unique observateur ayant eu voix fut la Convention de LaHaye qui prit la parole pour raconter les années de travail et d'études sur le thème de la juridiction de la consommation, lequel thème fut finalement mis de côté par la Convention suite à un plébiscite au sein de ce forum en 2005.
} 


\section{A) Revitaliser les CIDIPs : la réunion de Porto Alegre pour la CIDIP VII sur la protection des consommateurs et ses origines.}

\section{Un nouveau dialogue est possible entre la tradition civiliste et celle du Common Law au sein de l'OÉA}

La réunion préparatoire de Porto Alegre s'insère dans un cadre plus large d'efforts de codification du droit international privé au travers des CIDIPs et de revitalisation du système interaméricain ${ }^{7}$. S'inspirant de l'initiative étasunienne en matière de codification internationale du Droit international privé $e^{8}$, les CIDIPs sont les Conférences Spécialisées Interaméricaines sur le Droit International Privé organisées en tant que processus de codification depuis 1973, à l'échelle de l'OÉA (Organisation des États Américains) $)^{9}$, système au sein duquel furent déjà approuvés 26 textes internationaux ${ }^{10}$.

Depuis 1990, avec l'entrée dans l'OÉA du Canada ainsi qu'une participation plus active des Etats-Unis d'Amérique (ÉUA) ${ }^{11}$, ces Conférences spécialisées sur le Droit international privé suscitent davantage d'intérêt international ${ }^{12}$ car désormais, elle apportent, selon les mots de Jean-Michel Arrighi, «e défi de concevoir un droit interaméricain au sein duquel il serait possible d'associer le système juridique de type continental (civiliste) à celui de type anglo-saxon (Common Law) ${ }^{13}$.

Ce dialogue représente réellement un défi de taille, mais en est un de grande importance puisqu'il n'est pas souhaité que l'inclusion et la participation plus active ${ }^{14}$ de ces deux pays importants dans le système des CIDIPs viennent menacer ou fragiliser le système interaméricain si bien développé par l'OÉA durant son histoire.

7 Ainsi comme le démontrent FERNÁNDEZ ARROYO, Diego e MASTRÁNGELO, Fabio (Org.). El Futuro de la codifficación del Derecho internacional prizado en América- De la CIDIP VI a la CIDIP VIII, Org, Córdoba: Ed. Alveroni, 2005, p. 3 et suiv.

8 Voir à ce sujet: CASELLA, Paulo Borba e ARAUJO, Nádia (Coord.), Integraģäo Juridica Interamericana-As Convengöes Interamericanas de Direito Internacional Privado(CIDIPs) e o Direito Brasilearn, Ltr, São Paulo, 1998, et FERNÁNDEZ ARROYO, Diego. «La contribuición de la OEA al Derecho Internacional Privado", in Cadernos do Programa de Pós Graduagão em Direito-PPGDir. UFRGS, N. V, mars 2006, p. 231 et suiv.

- Voir FERNÁNDEZ ARROYO, Diego. La Codificación del Derecho Internacional Privado en América Latina- Ámbito de pmducción juridica y orientaciones metodológicas, Madrid: Eurolex, 1994, p 37 et suiv.

10 WILSON, in Cadernos do Programa de Pós-Graduação em Direito-PPGDir.UFRGS, N. V, mars 2006, p. 9. 21 documents sont em vigueur aujourd'hui, voir détails in www.oas org. ARRIGHI, Jean-Michel. OEA-Organizacäa dos Estados Americanos. Baurú:Manole, 2004 , p. 88 compile plus de 30 conventions. Jusqu’à maintenant, parmi les thèmes principaux des CIDIPs-OÉ $A$, on retrouve: lettres de change, les chèques, les preuves, mandat et représentation, sociétés mercantiles, preuves et informations du droit étranger, domicile des personnes physiques, normes générales de DIP, adoption de mineurs, contrats internationaux, trafic de mineurs, transport terrestre, garanties mobilières.

11 FERNÁNDEZ ARROYO, Diego. «La contribuición de la OEA al Derecho Internacional Privado», in Cadernos do Programa de Pós-Graduaşão em Direito-PPGDir./UFRGS, N. V, março 2006, p. 235

12 Voir: FERNANDEZ ARROYO, Diego (Org.). El Derecho internacional pricado interamericano en el umbral del siglo XXI, Ed. Eurolex, Madrid, 1997

${ }^{13}$ ARRIGHI, Jean-Michel. OE A-Organizą̧ão dos Estados Americanos. Baurú: Manole, 2004,p. 85. Voir aussi, au sujet des CIDIPs: OPERTTI BADAN, Didier, "Estado Actual del Derecho International Privado en ele Sistema Interamericano», IX Curso de Derecbo Internacional, vol. I, Secretaria General, OEA, 1983 et SIQUEIROS, José Luís. «Contribución de las CIDIP-I, II y III al Desarrollo del Derecho Intemacional Privadon, XIII Curso de Derecho Internacional, Secretaria General, OEA, 1987,p. 159-183.

${ }^{14}$ Soulignant la participation d'un institut prive de recherche étasunien pour la CIDIP V et VI (Centerfor Inter-American Free Trade, Tucson, Arizona), et sollicitant l'inclusion de thèmes davantage portés vers la protection de l'être humain et non seulement vers le commerce; FERNÁNDEZ ARROYO, Diego. Derecho Internacional Privado Interamericano. Buenos Aires: RubinzalCulzoni, 2000, p. 96 a 99 
Le danger de rendre inviable le système des CIDIPs peut émaner, selon certains, de deux raisons. D'abord, parce que les ÉUA et le Canada, des pays fédéraux, de tradition juridique du Common Law, auraient de la difficulté à négocier et ratifier des Conventions et pourraient ne prioriser, de façon radicale, que l'application de lois-modèles ou de soft laws, alors que le système interaméricain est davantage habitué aux efforts de codification par le biais de Conventions classiques de Droit international privé ${ }^{15}$. Ensuite, d'autres diront que le risque vient du fait que les thèmes et les modèles normatifs qui intéressent les pays que l'on désigne de la «famille juridique latino-américaine» (l'expression est de Diego Fernandez Arroyo $)^{16}$, comme, par exemple, la protection du consommateur plus vulnérable et la loi applicable aux contrats internationaux de consommation (proposition brésilienne pour la CIDIP-VII), n'intéresseraient pas les pays de tradition du Common Law.

Ainsi, selon certains, ces États, déjà fortement-industrialisés, ayant auparavant solutionné ces problèmes avec l'adoption de lois nationales, ne seraient pas intéressés par ces thématiques générales. De plus, les modèles normatifs qui intéresseraient les ÉUA et le Canada, comme, par exemple, l'arbitrage international de consommation (proposition étasunienne pour une des los-modèles pour la CIDIP VII) ou la juridiction avec la clause du forum non conveniens dans les causes impliquant la consommation (proposition canadienne de loi-modèle pour la CIDIP VII), de leur côté, n'intéresseraient pas les pays de la «famille juridique latino-américaine».

Comme l'ont montré les discussions de haut niveau lors de la préparation de la CIDIP VII, ce danger de rendre inviable le système interaméricain n'est, en fait, pas si grand. Selon l'opinion de la doctrine, le forum des CIDIPs et de l'OÉA, déjà habitué au dialogue juridique grâce à celui entre le système du Code de Bustamante et celui des traités de Montevideo de 1888-1889, semble être en condition pour s'affranchir de cette tâche ${ }^{17}$.

Tel que l'enseigne M. Erik Jayme, le dialogue ne peut s'initier, en ces temps post-modernes, que dans la conscience des différences et lorsque la pluralité des modèles est considérée en tant que valeur juridique ${ }^{18}$. En d'autres mots, le terme «dialogue »

15 La CIDIP I, à Panama en 1975 approuva 6 Conventions; la CIDIP II, à Montevideo en 1979, approuva 8 Conventions et Documents; la CIDIP III, à LaPaz en 1984, adopta 4 Conventions; la CIDIPIV, à Montevideo en 1989, adopta 3 Conventions; la CIDIP V, à Mexico en 1994, adopta 2 Conventions; mais la CIDIP VI, à Washington en 2002, à cause du protagonisme de ces deux pays, fut la première à ne voir l'adoption d'aucune Convention, on y adopta plutôt une loi-modèle et un «connaissement uniforme» (Uniforn Bills) sur le transport international. Voir: ARRIGHI, p. 88-89 e FERNÁNDEZ ARROYO, Diego e KLEINHEISTERKAMP, Jan. Die VI Interamerikanische Spezial Konferenz für Interantionales Privatrecht der OAS (CIDIP VI). Eine Marschroute interamerikanischen rechtlichen Integration, IPRAX 2002, p. 340 et suiv. Voir le résumé des CIDIPs dans SIQUIEROS, José Luis. «Reseña General sobre la quinta conferencia especializada interamericana sobre derecho internacional privado (CIDIP V)m, in CIJ-OEA, El Derecho Internacional Privado en las Américas (1974-2000), Cursos de Derecho Internacional - vol. I (Parte 1), Editor Secretaría General-Subsecretaria de Asuntos Jurídicos, Washington, 2002, p. 509-519.

16 FERNÁNDEZ ARROYO, Diego. Derecho Internacional Prizado Interamericano. Buenos Aires: Rubinzal-Culzoni, 2000, p. 11.

17 Tel que souligné dans FERNÁNDEZ ARROYO, in Cadernos do Programa..., p. 232-233.

18 JAYME, Erik. «Visões para uma Teoria pós-moderna do Direito Comparado», in Cademos do Programa de Pós Graduagão em Direito da Universidade Federal do Rio Grande do Sul-PPGDir.UFRGS, vol.1, n.1, mars 2003, p. 120, aussi publié dans Rerista dos Tribunais, vol. 759 , jan. 1999, p. 24-40. 
signifie deux logiques $(d i-a-\log o s)^{19}$, ce qui est le contraire de la mono-solution (monologue), et ne fonctionne véritablement que lorsqu'il y a «dialogue entre les différences». ${ }^{20}$

Dialogue sans vainqueurs et sans vaincus ${ }^{21}$, mais aussi entre formes de pensées différentes qui seront capables de négocier des positions cohérentes et possibles au sein de l'un et l'autre des systèmes, démontrant ainsi de véritables «vertus communicatives» ${ }^{22}$. Ceci implique, sans aucun doute, les thèmes et modèles législatifs qui rencontrèrent une réponse favorable parmi les 34 pays de l'OÉA plutôt que seulement parmi les pays les plus développés.

Erik Jayme ${ }^{23}$, dans son discours sur la vocation universelle du Droit international privé en janvier 2007 à la Conférence de LaHaye, fit l'éloge de cet effort que fait l'OÉA en affirmant:

«Au début du mois de décembre de l'an passé eût lieu, à Porto Alegre au Brésil, la conférence des spécialistes chargés de préparer la CIDIP VII, la septième Conférence spécialisée en Droit international privé. L'objet de cette conférence sera la protection des consommateurs dans le droit international privé, incluant les questions de compétence juridique et de la reconnaissance et l'exécution des sentences. Ceci est un fait notoire puisque cette conférence unit des pays de Common Law, tel les ÉUA,et les pays de tradition romano-germanique d'Amérique latine. De plus, ce sont des marchés comme le Mercosur qui ont besoin d'un droit international privé qui correspondrait aux exigences du marché. Dans certains pays d'Amérique latine, le droit international privé devient aussi un moteur pour la réforme des droits nationaux en matière de droits des consommateurs. Ils prennent en compte les développements européens, mais les solutions trouvées diffèrent. Alors que le droit européen en est à abandonner l'idée de l'autonomie de volonté des parties, les projets américains favorisent une combinaison entre le principe de la loi la plus favorable au consommateur et une forme d'autonomie limitée, solution que je vois moins radicale et plus équilibrée que le projet communautaire d'un règlement «Rome I»».

19 Voir LIMA MARQUES, Claudia. «Procédure civile internationale et MERCOSUR! pour un dialogue des règies universelles et régionales», in Rerue de Droit Uniforme, vol. III, 2003-1/2, UNIDROIT, p. 467 et suiv.

20 L'expression est des auteurs étasuniens BURBULES, Nicholas C. e RICE, Suzanne «Diálogo entre as diferenças: continuando a conversaçãom, in SILVA, Tomaz Tadeu da. (Org.). Teoria Educacional critica em tempos pós-modernos, Porto Alegre: Ed. Artes médicas, 1993, p. 173-204 (originalement publié dans Hariard Educational Revien, 61,4, 1991, p. 393-416).

24 Selon l'enseignement de BURBULES/RICE, p. 194, il est nécessaire de repenser au dialogue afin d'éviter qu'il ne devienne une «déroute» pour les partenaires plus faibles mais pour qu'il puisse plutôt créer un sentiment de «victoire» lorsqu'il y a compréhension des préoccupations de l'autre et permettre le développernent de (vertus communicatives» au sein des parties plus faibles comme des plus fortes

22 L'expression est de BURBULES/RICE, p. 197: «Le succès du dialogue entre les différences peut aussi dépendre de ce qu'on appelle les «vertus communicatives», lesquelles aident à tendre le dialogue possible et à entretenir une relation dialogique au fil du temps. Ces vertus comprennent la tolérance, la patience, le respect envers les différences, une disponiblilité pour écouter, l'humilité de pouvoir admettre que l'on peut ne pas avoir raison, la capacité de réinterpréter ou de traduire ses propres inquiétudes de façon à ce qu'elles deviennent compréhensibles pour les autres, l'auto-imposition d'une discipline de façon à ce que les autres puissent avoir la chance de parler ainsi que la disposition à s'exprimer honnêtement et sincèrement.»

${ }^{23}$ Discours pour l'occasion de l'inauguration d'un nouvel édifice à l'Académie de LaHaye, 18 janvier 2007, JAYME, Erik. A rocaģa universal do direito internacional privado - Tendências atuais, encore inédit. Traduction gracieusement cédée par Nádia de Araújo. 
Effectivement, la réunion à Porto Alegre a accentué la possibilité que la CIDIP puisse en arriver à créer un dialogue entre Common Law et tradition civiliste pour ainsi revigorer le système interaméricain, valoriser les CIDIPs antérieures tout en accentuant les expériences en matière de protection des consommateurs dans les pays de Common Law dans les Amériques. Cette possibilité de dialogue permettrait que soient approuvés le plus grand nombre possible d'instruments convergents, que ce soit des Conventions ou des Lois-modèles et ainsi revitaliser la CIDIP et l'OÉA en tant que forum de codification régionale, interculturel, effectif et se penchant sur des thèmes socialement importants, tel que la défense des consommateurs dans la société mondialisée.

Dans cette perspective, la réunion de Porto Alegre et les négociations qui la suivirent furent un grand succès, un dialogue sincère, honnête, créatif et fructueux dans la création d'un premier pas possible en matière de protection des consommateurs dans la région.

\section{Antécédents de la réunion préparatoire de Porto Alegre pour la CIDIP VII sur la protection des consommateurs}

La réunion de Porto Alegre pour la CIDIP VII sur la protection des consommateurs était la première à avoir lieu au Brésil, organisée par le gouvemement brésilien avec le partenariat d'une université publique (UFRGS, Porto Alegre) et une ONG (Brasilcon, Brasília) ${ }^{24}$. Cette réunion préparatoire reçut son lot d'éloges et fut considérée hautement fructueuse, ayant contribué effectivement à l'avancement des travaux préparatoires et à la consolidation des propositions à l'agenda des discussions pour la CIDIP VII, dont la réunion finale est prévue fin-200725.

Tant au sein du Forum virtuel, organisé par l'OÉA pour permettre la participation des délégués et d'experts dès mars 2006, que lors des réunions préparatoires, et parmi les 5 thèmes suggérés par le Comité juridique interaméricain en $2001^{26}$, les 8 thèmes suggérés pour la CIDIP VI en $2002^{27}$, les nombreux thèmes suggérés

24 Jusqu'à maintenant, les réunions préparatoires ainsi que les CIDIPs en tant que telles ont eu lieu à Panama (CIDIP I), en Uruguay (CIDIP II e IV), en Bolivie (CIDIP III), au Mexique (CIDIP IV), aux ÉUA (CIDIP VI), avec des réunions préparatoires dans ces mèmes pays, voir WILSON, John. «Introducción al Sistema Interamericano de Derecho Intemacional Privado: El proceso de la Séptima Conferencia Especializada Interamericana sobre Derecho Intemacional Privado (CIDIP VII) y la cooperación internacional em matéria de Derecho Internacional Privadon, in Cadernos do Programa de Pós-Graduagãa em DireitoPPGDir.UFRGS, N. V, mars 2006, p. 10-11.

25 Selon les propos formulés à la CAJP/OÉA du 18 janviet 2007

26 Voir «CIDIP-VII y Etapas sucesivas», CJI/doc. 74/01, avec les thèmes suivants: 1) Commerce électronique; 2) Migration et flux des personnes; 3)Arbitrage et resolutions alternatives des litiges; 4) Protection des consommateurs; et 5) Protection des mineurs, voir WILSON, p. 12 .

27 Voir CIDIP VI/RES. 01/02 et détails sur les thèmes considérés importants lors de la réunion de la CIDIP VI en prévision de la CIDIP VII, et qui incluaient le commerce électronique, PARRA-ARANGUREN, Gonzalo, "La sexta conferencia especializada interamericana sobre derecho internacional privado (CIDIP -VI, Washington, 2002)", in Rerista de Derecho (Tribunal Supremo de Justicia, Caracas, Venezuela), vol. 6, 
par les pays en $2003^{28}$, les 4 thèmes finaux du mandat de $2005^{29}$, desquels 2 ont été retenus pour l'élaboration d'instruments juridiques interaméricains pour la CIDIP VII (thèmes : 1 Protection des consommateurs et 2 - garanties mobilières : registres électroniques) ${ }^{30}$ et les 2 thèmes retenus pour l'élaboration d'études parallèles futures (thèmes: 1- Juridiction internationale, suggérée par l'honorable délégation de l'Uruguay dans le document «Bases de una Convención Interamericana sobre Junisdicción Internacionab〉 et 2-Registre des titres de propriété, suggéré par l'honorable délégation des ÉUA afin d'étendre les registres électroniques des garanties mobilières aux biens meubles et immeubles) ${ }^{31}$, il me semble très symbolique que seul le thème de la protection des consommateurs reçut un écho favorable de tous les pays.

En résumé, la protection des consommateurs fut le seul thème qui prospéra fortement durant ces 6 années (2001-2007), à tel point que l'Assemblée Générale et le Conseil permanent de l'OÉA décidèrent de séparer les thèmes et de consacrer la prochaine Conférence (VII) spécialisée à ce thème uniquement, ce qui permit un dialogue nord-sud au sein des Amériques.

Effectivement, en 2003, le Brésil ne fut pas seul à présenter une proposition pour la protection des consommateurs en Droit international privé («Convention interaméricaine sur la loi applicable à certains contrats et relations de consommation»). En effet, les deux pays les plus industrialisés des Amériques, le Canada et les ÉUA, appuyèrent l'idée que la CIDIP VII traite du thème de la protection des consommateurs par le biais du Droit international privé et présentèrent leurs propositions (Canadian Draft Proposal for a Model Law on Jurisdiction and Applicable Law et US Draft Proposal for a Model Inter-American Law on Avaliability of Consumer Dispute Resolution and Redress for consumers) ${ }^{32}$.

On discuta de ces trois textes, dans leurs versions révisées suite aux contributions du Forum virtuel, lors de la réunion de Porto Alegre. Le Brésil laissa aller sa langue portugaise et on discuta de la proposition brésilienne II entièrement en espagnol ( Propuesta de Convención Interamericana sobre la ley aplicable a algunos contratos y transacciones de consumo internacionales»). Notons que la proposition brésilienne II, sur laquelle on discuta durant la réunion de Porto Alegre, contenait déjà un préambule (lequel fut suggéré par l'honorable délégation d'El Salvador), 10 articles, plusieurs définitions et des clauses finales

28 Voir CP/CAJP-2094/03. Les thèmes envoyés étaient les suivants: «Pérou1. Transport : Dimension mondiale (terrestre, aérien, maritime); El Saliador 1. Uniformisation des titres universitaires: Libre exercice de la profession; 2. Responsabilité civile extracontractuelle: Accidents de la route; 3 . Responsabilité civile extracontractuelle: Produits; 4. Responsabilité civile extracontractuelle: Contamination environnementale; Brésil,1. Commerce électronique, 2. Insolvabilité commerciale transfrontière; 3. Mouvements transfrontaliers - Flux migratoires des personnes; 4. Protection des consommateurs: Convention interaméricaine pour la protection des consommateurs dans les Amériques; Mexique 1. Commerce électronique: Aspects juridiques de l'usage des technologies, Protection des consommateurs, 2. Mouvements transfrontaliers - Flux migratoires des personnes; 3. Protection des mineurs; Canada 1. Commerce électronique: Questions juridictionnelles reliées à la protection des consommateurs; Uruguay 1. Juridiction internationale; 2. Responsabilité civile extracontractuelle: Contamination environnementale; 3 . Commerce électronique: Juridiction en matière de transactions transfrontalières par Internet entre entreprises et consommateurs; Etats-Unis 1. Commerce électronique: Valeurs des investissements; 2. Commerce électronique: Registre commerciaux électroniques.»

2 Voir AG/RES 2065 XXX-O/05.

30 Pour plus de détails, voir WILSON, p. 13-16

31 Pour plus de détails, voir WILSON, p. 16

2 Voir les propositions: www.oas.org. Et pour plus de détails sur celles-ci, voir WILSON, in Cadernos do Programa de Pós-Graduagãa em Direito-PPGDir/UFRGS, N. V, mars 2006, p. 28-31 
(suggérées par les honorables délégations d'Argentine, d'El Salvador, du Paraguay, d'Uruguay et du Mexique ainsi que par les observateurs invités de la UNCITRAL et du Canada). Mais les discussions se concentrèrent sur les 7 articles principaux (art.1. Définition de consommateur, Art. 2. Protection contractuelle générale : détermination de la loi applicable, Art. 3. Normes impératives, Art. 4. Clause d'exception et harmonisation, Art. 5. Thèmes exclus, Art. 6. Contrats de voyage et de tourisme, Art. 7 . Contrats de multipropriété-timesharing). ${ }^{33}$

\section{B) Avancées et discussions principales lors de la réunion de Porto Alegre pour la CIDIP-VII sur la protection des consommateurs}

La réunion de Porto Alegre fut ouverte par plusieurs belles interventions des représentants du gouvernement brésilien, de l'OÉA, de la UFRGS ainsi que par un discours d'ouverture du président de Consumers International de Londres. Cette ONG mondiale, auparavant dénommée IUCO, réunit aujourd'hui plus de 300 associations de défense des consommateurs de 105 pays différents. Sa présidente mondiale, la Brésilienne Marilena Lazzarini, mit l'accent sur l'importance de cette réunion pour le futur de la défense des consommateurs dans la région:

«En ce moment, je peux me permettre de dire que les consommateurs des Amériques et du monde entier mettent beaucoup d'espoir dans cette réunion préparatoire et sur le projet de Convention interaméricaine qui, je le pense, devrait en émaner. Il est nécessaire d'établir une borne internationale en protection des consommateurs. Une réponse régionale qui répond à des questions universelles! Un premier pas sûr et important dans la direction de l'établissement de normes internationales de protection des consommateurs! Des normes effectives qui aideront les pays qui ne possèdent toujours pas de normes sur le commerce international, des normes qui symboliseront un compromis pour la protection effective des consommateurs touristes, des consommateurs bombardés quotidiennement par une publicité agressive et séductrice des produits et de services étrangers, des normes qui créeront une confiance envers le commerce électronique ou à distance en constante croissance. (...) Je perçois ce moment comme une marque internationale pour la protection des consommateurs, et je vois en chacun de vous ici présents une disposition naturelle pour assumer la responsabilité de confirmer cette marque à Porto Alegre, au Brésil, la faisant ainsi résonner sur toute l'Amérique et les autres continents. Une réponse qui, sans aucun doute, sera osée, courageuse et engagée envers un changement et un avancement effectifs des législations de protection des

33 Voir le texte de la seconde proposition brésilienne sur le site de l'OÉA, www.oas.org ou dans l'article, LIMA Marques, Claudia. «A proposta brasileira de Convenção Interamericana de Direito Internacional Privado sobre lei aplicável a alguns contratos com consumidores (CIDIPVII): temas e discussões no Fonum de expertos da OEA», in Cadernos do Programa de Pós-Graduação em Direito-PPGDir./UFRGS, N. V, mars 2006, p. 75-106 
droits humains des consommateurs! Que cette réunion à Porto Alegre au Brésil soit le symbole que l'OÉA et ses 34 pays membres considèrent que la protection des consommateurs dans les contrats internationaux est un pas nécessaire pour construire un monde meilleur, plus harmonieux et juste au sein du marché mondialisé de la consommation!»

Ensuite, fut lu un message du Professeur Iain Ramsey de l'Université York (Toronto, Canada $)^{34}$, président mondial de la International Association of Consumer, une association de professeurs et de juges spécialisés dans la défense des consommateurs.

Après la cérémonie officielle, laquelle fut enrichie par une belle intervention de Jean-Michel Arrighi, s'initia la session fermée et Dr. Ricardo Morishita Wada, directeur du Département de protection et de défense des consommateurs du Ministère de la Justice-SDE, fut élu président et chef de la délégation brésilienne. Une fois l'agenda accepté, les discussions au sujet des trois propositions pour la CIDIP-VII sur la protection des consommateurs s'initièrent.

\section{Les discussions sur les propositions}

Lors de la réunion de Porto Alegre, on discuta au sujet de trois textes durant six heures pour chacun d'eux ${ }^{35}$ en commençant par le texte brésilien, ensuite celui des étasuniens pour terminer avec le canadien.

Le projet brésilien de Convention sur la loi applicable aux contrats internationaux de consommation fut bien reçu lors de la réunion. On a discuté sur la totalité du texte et le projet fut considéré prometteur. Aussi, il fut décidé qu'il devait être soumis à un groupe de travail de rédaction car plusieurs de ses normes furent reformulées durant la réunion. Ainsi, furent inclus des articles clarifiant le test de la loi la plus favorable et de nouvelles définitions afin de favoriser la ratification de cette future Convention par un plus grand nombre de pays. L'acte final de la réunion de Porto Alegre affirme que «...on arriva à un accord général sur plusieurs thèmes - en dépit des préoccupations spécifiques envers certains aspects liés à la rédaction des articles. À cette étape, on n'a pas adopté le système des crochets dans le texte; à la place, les participants ont plutôt

\footnotetext{
${ }^{34}$ Le texte envoyé et lu au public par le directeur de Brasilcon allait comme suit: «... I am sorry that I am unable to attend this important meeting of the Inter-American Conference on Prizate International Lam. Globalization and the spread of the internet mean that consumers increasingly shop across borders. This has benefits for consumers and businesses but consumers may face significant burdles in achieing redress in the event that there are problems with their purbases. The barriers facing consumer redress at the national level are well knonn and these are multiplied in cross-border transactions. It is zital therefore that rules on jurisdiction and choice of lan' should be modernized in consumer transactions to reflect the realities of these power imbalances in cross-bonder transactions. The work of the Inter-A merican Conference is very nelomme in this respect and the Brazilian proposals for this meeting provide a valuable template for a convention that mill promote consumer confidence in oross-bonder transactions. I bope that you baie a stimulating and productive meeting. Best regards, Iain Ramsay - President, International Association of Consumer Lan; Professor of Lan; Orgoode Hall Lan School, Tononton

${ }^{35}$ Durant la réunion, tous les articles de ces trois projets furent discutés, à la seule exception de la seconde partie du Projet Canadien sur la loi applicable aux contrats de consommation (section 7) qui ne put être débattue une fois que les délégations manifestèrent des objections générales à son sujet et qu’elles soulignèrent qu'elle est incompatible avec la proposition brésilienne. Aussi, les discussions portant sur les articles de la première partie de la proposition canadienne, furent très longues et très courues et amenèrent plusieurs interventions et critiques en raison de l'existence du projet uruguaien d'études parallèles pour la CIDIP VII sur les «bases d'une Convention interaméricaine sur la juridiction intetnationale».
} 
cherché à arriver à un consensus sur les idées et les forces du projet, et ils effectuèrent des observations ponctuelles au sujet des normes. On s'est entendu sur le fait que la rédaction sera perfectionnée par un groupe de travail organisé par l'OÉA, en accord avec l'agenda qui devra être adopté, afin de permettre que le texte final soit ratifié par le plus de pays possible.» ${ }^{36}$

Suivit ensuite la discussion à propos du projet étasunien, lequel fut bien reçu et qui, lui aussi, devrait passer par un groupe de travail de rédaction ${ }^{37}$. Le texte fut considéré large et encore à son étape de rédaction conceptuelle, mais aussi prometteur : «Les délégations exprimèrent leur appui au projet en général, incluant les actions en réparation individuelles, collectives et gouvemementales. Aussi, elles soulignèrent l'interaction de la proposition avec plusieurs autres thèmes tels les contrats, les actes illicites, le droit pénal, le droit de procédure interne et le droit de procédure international. Quelques-uns de ces points furent discutés et d'autres le seront ultérieurement. On suggéra aussi d'améliorer le texte et la rédaction ainsi que l'ajustement de certains points afin de maintenir l'intention positive, vue la diversité des droits de procédure et des cultures existantes entre les pays de l'OÉA, d'encourager la protection des consommateurs à travers des mécanismes d'accès à la justice.».

36 (en espagnol dans le texte original) L'acte final affirme, sur le projet brésilien : «Le projet en général fut bien reçu. Les délégués adhèrèrent aux objectifs de la proposition brésilienne : offrir une protection légale pour les consommateurs dans leurs relations avec les foumisseurs, foumir les bénéfices économiques aux consommateurs en augmentant la disponibilité et en diminuant les coûts des produits et offrir confiance aux consommateurs envers le marché. Ces antécédents furent considérés durant l'analyse du texte et on arriva à un accord général sur plusieurs thèmes - en dépit des préoccupations spécifiques envers certains aspects liés à la rédaction des atticles. À cette étape, on n’adopta pas le système des crochets dans le texte; à la place, les participants ont plutôt cherché à arriver à un consensus sur les idées et les forces du projet, et ils effectuèrent des observations ponctuelles sur les normes. On s'entendit sur le fait que la redactyion sera perfectionnée par un groupe de travail organisé par l'OÉA, en accord avec l'agenda qui devra être adopté, afin de permettre que le texte final soit ratifié par le plus de pays possible. On exprima l'opinion que les règles de la proposition sont en générales positives mais qu'il faudrait considérer ses interactions en prenant en compte que l'objectif de la Convention de droit intemational privé est de déterminer quel doit être le droit applicable. En guise de résumé, les aspects de la rédaction qui devront être considérés par le groupe de travail de rédaction sont les suivants : - La définition de contrat de consommation à l'article 2.1. - l'utilisation, la portée et la définition des règles impératives et de l'application de l'ordre public à l'art. 3. - les provisions de la clause de l'Art. 4. - Les aspects de rédaction des Ast. 5 à $7 . »$

37 Le texte de l'acte final va comme suit (traduction libre): «Durant le deuxième jour de la réunion, on débattit en premier lieu de la proposition des États-Unis. La délégation étasunienne fit quelques commentaires au sujet de sa proposition et suivirent ensuite les commentaires de plusieurs délégations qui appuyaient les objectifs de la proposition étasunienne. Aucune emphase spéciale ne fut mise lors de cette étape sur la rédaction du texte. Les délégations étaient d'accord avec le fait que la proposition étasunienne et la proposition brésilienne sont complémentaires et ne s'excluent pas mutuellement. Ensuite, une discussion article par article eût lieu lors de laquelle la délégation étasunienne émit ses commentaires généraux sur chacun d'eux. Les délégations exprimèrent leur appui au projet en génétal, incluant les actions en réparation individuelles, collectives et gouvernementales. Aussi, elles soulignèrent l'interaction de la proposition avec plusieurs autres thèmes tels les contrats, les actes illicites, le droit pénal le droit, le droit de procédure interne et le droit de procédure international. Quelques-uns de ces points furent discutés et d'autres le seront ultérieurement. On suggéra aussi d'améliorer le texte et la rédaction de même que l'ajustement de certains points pour maintenir l'intention positive d'encourager la protection des consommateurs à travers des ces mécanismes d'accès à la justice vue la diversité des droits de procédure et des cultures existantes entre les pays de l'OÉA. Aussi, il faudra considéter une référence à des définitions existantes au sein des ordres internes afin d'assurer sa compatibilité. La section relative aux solutions de litiges et de réparations gouvernementales fut considérée comme un aspect positif. En même temps, plusieurs exprimèrent l'opinion qu'il faudra une meilleure connaissance des détails de ces dispositions avant de procéder à leur inclusion dans la loi-modèle. Une délégation fit une référence spécifique au thème des cartes de crédit. Il y eût consensus général sur la portée et l'objectif général, y compris les litiges, tant au niveau interne qu'intemational, et la nécessité de prévoir des mécanismes permettant une réparation adéquate. En général, le projet fut bien reçu, en dépit du fait que la rédaction devra être améliorée par le groupe de travail proposé.» 
Ainsi, si dans les deux premiers jours de la réunion, les délégations et les experts présents soulignèrent la nécessité d'améliorer les deux textes, ces délégations firent ressortir que ces textes, brésilien et étasunien, peuvent servir de base pour la future CIDIP VII. En effet, ces textes sont en convergence au niveau des thèmes et des objectifs, n'entrainent aucune exclusion ou incompatibilité entre les normes proposées soit de la Convention, soit de la loi-modèle, et ainsi, on peut lire dans l'acte final: «Les délégations étaient d'accord avec le fait que la proposition étasunienne et la proposition brésilienne sont complémentaires et ne s'excluent pas mutuellement.»

Finalement, fut débattu également durant six heures le projet canadien en général et sa première partie sur la juridiction dans la consommation. L'acte final relate que «Les participants procédèrent ensuite à la discussion au sujet de la proposition canadienne sur la loi-modèle de juridiction et de la loi applicable. Celle sur la loi applicable est un complément au texte initial qui fut préalablement présenté par la délégation canadienne. Les délégués reçurent favorablement la présentation et considérèrent que le thème est très important.»

Quant à la proposition canadienne, les interventions des délégations à son sujet furent très divisées et il y eût plusieurs manifestations d'inquiétudes, de questionnements et de commentaires. En fait, il y en eût tant que l'article unique de la deuxième partie du Projet canadien sur la loi applicable aux contrats de consommation (partie 7) ne put être débattu durant la réunion, faute de temps. ${ }^{38}$

Notons que l'expérience de la Conférence de LaHaye, dont un représentant était présent à la réunion de Porto Alegre, fut relatée. Ces 10 années de discussions infructueuses sur le thème de la juridiction dans les litiges de consommation entre la tradition civiliste et celle du Common Law furent mises en évidence, créant ainsi des doutes sur la capacité des experts et des délégués de l'OÉA à en arriver à un accord qui serait le meilleur modèle des Amériques sur la juridiction (nationale et internationale) de la consommation afin d'ainsi approuver une loi-modèle interaméricaine. De plus, le fait qu'actuellement dans le système interaméricain il existe déjà un projet uruguayen en études parallèles pour la CIDIP VII nommé «Bases pour une Convention interaméricaine sur la

39 Dans l'acte final, ces débats sont ainsi résumés : «Les questions et les inquiétudes envers la proposition incluaient les suivantes : - On suggéra la réalisation de travaux additionnels sur les définitions contenues dans la proposition. Quelques délégations ont indiqué qu'il serait souhaitable d'inclure des définitions dans le texte du document. - On fit remarquer que la rédaction pourrait être améliorée. Entre autres choses, certains exprimèrent qu'il serait préférable de recommencer la rédaction de l'article 3 et, en particulier, y inclure une référence à l'article 6 qui exige qu'un tribunal refuse d'exécuter une clause d'élection du forum en certaines circonstances. - Fut aussi exprimée l'inquiétude envers l'inclusion du forum non conveniens dans le brouillon de la proposition. D'autres opinions futent émises à l'effet qu'il serait nécessaire d'inclure le forum non conteniens à la proposition. Des inquiétudes furent formulées sur la viabilité générale de la règle de l'article 4 en termes de son application au commerce électronique, tout comme de la charge que cette disposition impose au vendeur de prendre tous les moyens raisonnables pour éviter de contracter avec des consommateurs résidants à l'étranger. Aussi fut manifestée l'inquiétude sur le fait que cette charge serait particulièrement onéreuse pour les petites et moyennes entreprises et qu'il faudrait reconnaître l'autonomie des parties. - Aussi on exprima des commentaires sur le fait que la proposition devrait prendre la forme d'une convention et non d'une loi-modèle. Des opinions dans le sens inverse furent aussi formulées. - On prit position sur l'interrelation entre cette loi-modèle et les propositions étasunienne et brésilienne. - Furent considérées les inquiétudes sur l'application de la proposition aux contrats internes de consommation et sur la présomption que les normes internes devront être modifiées en conformité avec la loi-modèle.» 
juridiction intemationale » est venue amplifier ces doutes, car cette dernière pourrait très bien inclure le thème de la consommation et les préoccupations de la proposition canadienne.

Quant aux détails de la proposition canadienne, tous les pays latino-américains présents à la réunion exprimèrent leurs inquiétudes envers l'usage d'une loi-modèle (pour tous les contrats de consommation, nationaux comme internationaux) plutôt que d'une Convention, comme celle déjà proposée par l'Uruguay. Aussi, ils manifestèrent leurs inquiétudes quant au fait de traiter de deux thèmes aussi importants que la juridiction et la loi applicable, lesquels impliquent les droits humains, à l'intérieur d'un unique document. De plus, ils soulignèrent fortement l'incompatibilité entre le modèle de la deuxième partie sur la loi applicable de la proposition canadienne et la proposition brésilienne pour une Convention ainsi qu'entre la première partie du projet canadien avec la proposition étasunienne, lesquelles furent déjà discutées et bien reçues durant la même réunion.

Plus spécifiquement, une forte inquiétude fut causée par la présence du forum non conveniens (discrétion du juge compétent à accepter d'entendre la cause) dans les recours intentés par les consommateurs, principe méconnu par le Droit latinoaméricain. Le forum non conveniens fut considéré par certains comme étant une négation de juridiction et de l'accès du consommateur à la justice (droit humain et fondamental), c'est sur ce sujet que les débats furent longs et forts courus.

Cette réaction plus forte envers la proposition canadienne durant la réunion de Porto Alegre peut avoir comme fondement le fait que cette proposition semble émaner d'une position doctrinaire et philosophique différente des propositions brésilienne et étasunienne. Ces deux proposition valorisent l'autonomie des parties en Droit international privé et cherchent des façons de concilier la protection du consommateur et l'autonomie des parties, validant ainsi les clauses d'élection de la loi (dans le cas de la proposition brésilienne) et - indirectement - du forum (arbitrage, dans le cas de la nouvelle proposition étasunienne) dans les contrats de consommation. Aussi, elles encouragent la croissance du commerce dans la région en combattant les pratiques actuelles de discrimination des consommateurs qui ont résidence ou domicile dans certains pays déterminés (cette pratique est permise et validée dans la proposition canadienne). La proposition canadienne opte pour la régulation et prévoit l'invalidité des clauses d'élection de la loi et du forum dans les contrats d'adhésion pour les consommateurs, sanctionnant aussi par l'invalidité plusieurs cas régulés dans les deux autres propositions, lesquelles furent discutées avant et bien reçues à la réunion. Cette incompatibilité initiale de philosophies différentes peut avoir été un des problème qui a suscité cette inquiétude majoritaire envers la proposition canadienne. De plus, la façon dont la proposition canadienne est rédigée n'est pas habituelle pour les pays de tradition civiliste et pourrait avoir été mal comprise par les pays latino-américains, bien que ceci ne réussit pas à expliquer la forte réaction de l'honorable délégation étasunienne envers la proposition canadienne.

Par contre, notons que le Canada est l'un des pays ayant la plus grande expérience en matière de protection des consommateurs dans les contrats internationaux (spécialement dans le commerce ÉUA-Canada), et de tout temps il fut 
un pionnier dans ce domaine car, justement, il sut profiter de l'expérience du dialogue entre tradition civiliste et tradition du Common Law survenant de son commerce interne. Dans ce sens, il reste deux perspectives. Celle où la proposition canadienne pourrait avoir une nouvelle version, comme les autres propositions, et être ainsi considérée pour la CIDIP VII. Ou encore, que les préoccupations de la doctrine canadienne sur la validité des clauses d'élection de la loi applicable dans les contrats d'adhésion de consommation aillent influencer les autres propositions, en particulier la brésilienne, si le projet canadien était retiré ou inclus en tant qu'étude parallèle pour les prochaines CIDIPs tel qu'il le fut suggéré lors de la réunion.

\section{Groupe de travail et de rédaction informel : les négociations ultérieures à la réunion de Porto Alegre}

Conformément à la méthode de travail déterminée, la réunion de Porto Alegre fut en effet suivie de plusieurs négociations informelles. Durant cette réunion, il fut établi qu'un "groupe de travail de rédaction» allait élaborer la version finale des documents. Mais, à cause d'un problème quant aux coûts que cela aurait engendrés, le groupe de travail resta informel (courriels, textes, documents officiels, téléconférences, messages textes, rencontres informelles) et travailla la plupart du temps à distance. L'aspect positif est que, suite à la réunion de janvier 2007, il était maintenant possible d'inclure les délégués des 11 pays présents à la réunion de Porto Alegre une fois qu'ils avaient tous envoyé des collaborations et ainsi, il ne fut pas nécessaire de rouvrir le Forum Virtuel des discussions alors que plusieurs contributions avaient déjà été faites.

Dans le cas de la proposition brésilienne, nous avons reçu par écrit après la réunion de décembre, plusieurs suggestions et spécialement des corrections linguistiques de la part des délégués et des experts d'Argentine, d’Uruguay, du Paraguay et d'El Salvador, ce dont je tiens à les remercier. Lors de la réunion de la CAJP-OÉA le 18 janvier 2007 à Washington, cette méthodologie fut consolidée, selon laquelle la nouvelle version de la proposition brésilienne serait envoyée aux 11 pays présents à Porto Alegre avant le 31 mars 2007, ce qui fut fait (Propuesta de la Delegación Brasileña- III, de 12 de Deciembre 2006, Propuesta de Convención Interamericana sobre la ley aplicable a algunos contratos y transacciones de consumo internacionales). De plus, les ÉUA ont envoyé une nouvelle version de loi-modèle générale pour une nouvelle ronde de critiques et suggestions qui s'étendait jusqu'au 30 avril.

S'ensuivirent donc plusieurs nouvelles et fructueuses suggestions, critiques et listes de cas hypothétiques venant de presque tous les pays, incluant des collaborations du Canada et des ÉUA. Cette méthode de travail qui fut aménagée lors de la réunion de CAJP de janvier 2007 , lors de laquelle on affirma que, pour éviter les dépenses, les délégués devraient continuer à avoir recours aux envois de courriels, aux téléconférences, aux rencontres informelles lors de congrès ou de cours de l'OÉA, aux visites spontanées de courtoisie et même aux vidéoconférences de façon à ce que tous puissent participer et collaborer à l'élaboration de la version finale des propositions, ce qui fut fait. C'est de cette façon que le délai pour l'envoi 
des collaborations écrites, initialement prévu pour le 30 avril 2007, fut repoussé jusqu'à la rencontre au Paraguay en octobre 2007. C'est donc alors que la version finale de la proposition brésilienne sera présentée et envoyée aux autres pays de l'OÉA.

De plus, notons que lors de la réunion de Porto Alegre, l'honorable délégation des ÉUA manifesta une inquiétude particulière envers la règle sur la présomption de la loi la plus favorable au consommateur. Ainsi, cette norme fut modifiée -à la demande des ÉUA et ce, durant la réunion même - par la délégation brésilienne afin qu'elle ne contienne plus une présomption mais plutôt une liste d'indicatifs de la loi la plus favorable (art. 2, 3 Propuesta Brasileña III). Durant les négociations ultérieures, on travailla sur l'amélioration de cette règle, en regroupant quelques-unes des suggestions des honorables délégations du Canada et de ÉUA, mais on n'arriva pas à un plein consensus sur le sujet, ce qui devrait donc être réservé pour la discussion lors de la réunion finale pour la CIDIP VII. De même, le thème de la possibilité d'émettre des réserves à la future Convention est réservé pour la réunion finale.

Finalement, nous remarquerons que la délégation brésilienne, reconnaissant l'importance des préoccupations contenues dans le projet canadien de loi-modèle, incorpora volontairement dans la nouvelle version de son projet de Convention (art. 4, 2bb Propuesta Brasileña III) une option basée sur le texte de l'article 7 de la proposition canadienne avec quelques modifications au deuxième paragraphe de son article sur les normes impératives et la fit parvenir aux 11 pays présents à la réunion.

Cette nouvelle option doit être maintenue dans le texte final brésilien qui sera présenté au Paraguay en octobre 2007 malgré le fait que quelques délégués s'étaient affichés contre l'option au sein du groupe de travail informel. À ce sujet, l'idée de la délégation brésilienne est que, en maintenant cette option d'invalidité de la clause d'élection de la loi tout en la réduisant au thème d'irrespect des normes impératives (et non de toutes les lois) du pays du domicile du consommateur (ou de sa résidence habituelle, telle que formulé dans la proposition canadienne), les délégués pourront choisir, lors de la réunion finale pour la CIDIP VII. Le choix se fera donc entre la norme originale brésilienne prévoyant la cumulation de la lex contractus choisie par les parties avec les normes impératives du pays du domicile du consommateur et la solution canadienne de l'invalidité totale de la clause d'élection de la loi si celle-ci est incompatible avec les normes impératives du domicile du consommateur selon ce que les délégués considèreront être la meilleure solution pour la protection des consommateurs dans la région.

La proposition européenne de Réglementation mentionnée, appelée «Rome I» par le professeur Erik Jayme et qui doit remplacer la Convention de Rome de 1980, remplace d'une certaine forme l'autonomie des parties (connexion subjective) par une connexion objective et unique. En effet, elle impose l'application systématique de la loi du pays de la résidence habituelle du consommateur, ce qui entraine donc l'invalidité des clauses d'élection du forum. Cette solution de Rome I, comme nous pouvons l'observer dans l'intervention d'Erik Jayme, est aussi très critiquée en Europe, mais elle pointe vers un degré encore plus élevé de protection des consommateurs dans ce marché intégré économiquement. 
La délégation et les experts brésiliens manifestèrent de forts doutes à savoir si cette position de la proposition canadienne est la meilleure et doit être le «modèle de codification» du futur dans un marché non intégré tel que celui des Amériques car elle exige la modification des lois des 33 autres pays de l'OÉA, d'autant plus qu'elle vise aussi les contrats internes de consommation. Mais en tant qu'option possible en matière d'incompatibilité avec les normes impératives de l'État du domicile du consommateur dans les contrats internationaux dans lesquels ces consommateurs sont passifs (par exemple, dans le commerce électronique), et dans l'esprit général du respect de l'autonomie limitée des parties de la proposition brésilienne, cette nouvelle option peut être utile au dialogue entre tradition civiliste et tradition du Common Law.

De plus, les ÉUA ont décidé d'élargir leur proposition. Ils ont donc envoyé dernièrement aux 11 pays, pour examen et suggestions, la proposition originale de loi-modèle, maintenant transformée en Lignes directrices (Legislative Guide on Consumer Redress) sous la suggestion de l'honorable délégation argentine, ainsi que trois nouvelles lois-modèles. Celles-ci sont totalement nouvelles et développent quelques concepts de la loi-modèle générale auparavant présentée lesquels sont 1) Model Law on Redress for small monetary claims, 2) Model Law for electronic arbitration of cross border claims et 3) Model Law on government authority. ${ }^{39}$

Enfin, il est pertinent de mentionner que le Canada a envoyé jusqu'à maintenant le même texte qui fut débattu à Porto Alegre aux 11 pays présents à la réunion. Mais la délégation déclara lors de la réunion de la CAJP-OÉA de janvier 2007, qu'elle était en train de travailler sur une nouvelle version encore non diffusée.

Ceci étant, nous pouvons affirmer sans l'ombre d'un doute que les travaux de suivi de la réunion de Porto Alegre de décembre 2006 pour la préparation de la CIDIP VII sur la protection des consommateurs ont été très fructueux. Ces travaux informels furent finalisés seulement en octobre 2007.

Ainsi, la réunion de Porto Alegre permit un avancement significatif pour les propositions étasunienne et brésilienne, les modifiant et les faisant avancer significativement en direction des textes finaux maintenant débattus. De cette réunion et des négociations qui la suivirent pour la préparation des textes définitifs, nous pouvons tirer plusieurs leçons, c'est ce que j'analyserai maintenant.

\section{Les consensus de la réunion de Porto Alegre et des négociations informelles du Groupe de travail et de rédaction pour la CIDIP VII sur la protection des consommateurs}

En tant que simple professeure de Droit international privé et témoin de ce processus initié il y a plus de six ans - et non en tant que déléguée brésilienne -, je crois qu'il est possible de tirer quelques leçons de ce long chemin parcouru. Je pense qu'aujourd'hui, il

3 Voirwww.oas.org. 
existe un consensus dans la région sur l'opportunité de codifier dans la CIDIP VII la protection des consommateurs.

Effectivement, à partir de ma première suggestion lors du cours de l'OÉA en 2000, en passant par les 5 thèmes suggérés par le Comité juridique interaméricain en 2001 incluant la protection des consommateurs ${ }^{40}$, l'acceptation du thème du commerce électronique à l'agenda de la CIDIP VII en $2002^{41}$, les recherches sur les thèmes et la convocation des experts pour la CIDIP VII en $2003^{42}$ et en $2004^{43}$, la réduction à deux thèmes pour la future CIDIP VII par l'Assemblée Générale de l'OÉA ${ }^{44}$, le commencement des travaux préparatoires du groupe d'experts en mars $2006^{45}$, l'institution du très utile Forum Virtual-OEA de discussions entre tous les délégués et experts sous l'organisation et la modération de John Wilson (OÉA) ${ }^{46}$, la réunion préparatoire de Porto Alegre en décembre 2006, jusqu'à septembre 2007 alors que nous avons finalisé les textes, plus de six années de discussions et de négociations se sont passées.

Dans ce sens, mon témoignage consiste à dire que les leçons à tirer doivent avoir une relation directe avec les deux grands thèmes qui ont été discutés durant ce processus, c'est-à-dire:

1) De la nécessité de normes spéciales de défense des consommateurs en matière de contrats internationaux de consommation au sein du système interaméricain vu la distinction entre la situation de vulnérabilité du consommateur face au fournisseur, plus particulièrement dans le commerce électronique (B2C) et la situation plus équilibrée du commerce international entre deux professionnels (B2B), déjà régulée par la CIDIP V de Mexico de 1994 (ici peut être incluse la grande discussion sur l'inclusion ou non des personnes juridiques dans la définition du consommateur à protéger par la CIDIP VII et si celle-ci devrait réguler ou inclure des règles sur la responsabilité pour les produits défectueux) et;

2) Des instruments à choisir pour réaliser cette opportune protection des consommateurs en Droit international privé dans la région, à savoir s'il serait plus adéquat d'opter pour une Conventions classique de Droit international privé (Hard Law), dont la dernière du genre est la CIDIP V de 1994, laquelle est, treize ans plus tard, ratifiée en 2007 seulement par le Mexique et le Venezuela; ou encore s'il serait plus souhaitable que cette

40 CIDIP-VII E Etapas sucesizas CII/doc. 74/01

${ }^{41}$ CIDIP VI/Res.1/02. Voir aussi PARRA-ARANGUREN, Gonzalo. «La sexta conferencia especializada interamericana sobre derecho internacional privado (CIDIP -VI, Washington, 2002)», in Revista de Derecho (Tribunal Supremo de Justicia, Caracas, Venezuela), vol. 6, p. 275.

${ }^{42} \mathrm{AG} /$ Res. $1923, \mathrm{XXXII-O} / 03$

${ }^{43} \mathrm{AG} /$ Res.2033, XXXIV-O/04

${ }^{44}$ Les deux thèmes sont: protection des consommateurs et garanties mobilières : registres électroniques pour l'implémentation de la loi-modèle interaméricaine sur les garanties mobilières. AG/RES 2065, XXX-O/05

${ }^{45}$ AG/RES 2217, XXXVI-O/06

${ }^{46}$ WILSON, in Cadernos do Programa de Pós-Graduạ̧ão em Direito-PPGDir./UFRGS, N. V, março 2006, p. 28-31. 
protection soit assurée grâce à des loi-modèles et des lignes directrices (Soft Law), dont la dernière (et unique) de ce genre est de 2002 et, durant ces cinq années, peu de succès ont pu être démontrés; et à savoir quelle sera la portée d'application de ce nouvel instrument interaméricain de protection des consommateurs (s'il ne concernera que le droit applicable, s'il inclura ou non une juridiction spéciale du consommateur, s'il traitera seulement des thèmes spéciaux de procédure civile internationale, administrative et pénale, comme les compensations monétaires, les tribunaux de petites créances, l'arbitrage de consommation, les actions gouvernementales et la coopération entre agences, les actions collectives privées et des associations, etc.).

Il est intéressant d'observer que si la réunion de Porto Alegre connût du succès, ce fut justement parce qu'elle put éclairer les délégués représentés sur le fait que le terrain était déjà prêt pour atteindre un consensus sur ces thèmes. S'il ne fut pas atteint de manière totale, il le fut au moins de manière initiale puisque le dialogue sur la protection des consommateurs à l'OÉA en est encore à ses débuts et la CIDIP VII marque son commencement plutôt que sa fin. Voyons cet aspect plus en détails.

\section{A) Consensus général sur la nécessité et sur l'opportunité d'élaborer des normes spéciales de droit international privé pour la protection des consommateurs au sein de l'OÉA : la protection du consommateur en tant que lacune dans le système interaméricain}

Le plus important résultat de la réunion de Porto Alegre et des négociaticons ultérieures, à mon opinion, fut d'atteindre un consensus au sujet de l'occasion qui se présente d'inclure à l'agenda de l'OÉA la protection des consommateurs dans la région.

Lorsque je fus invitée par Jean-Michel Arrighi à réfléchir sur la protection des consommateurs dans les Amériques durant mon cours à l'OÉA en 2000 (La protection du consommateur: aspects de droit privé régional et général), j’ai identifié une lacune dans le système interaméricain de Droit international privé (DIP) en matière de protection des consommateurs et la nécessité d'établir des normes spéciales en matière de conflits de lois puisque ces dernières n'étaient considérées suffisantes qu'au Canada et aux ÉUA. Ainsi donc, j’ai reconnu l'occasion d'élaborer une Convention interaméricaine spécialement sur le thème des contrats de consommation, englobant les contrats issus du commerce électronique ainsi que certains contrats complexes de tourisme de masse, de façon à augmenter le niveau de protection des consommateurs dans tous les pays membres de l'OÉA. ${ }^{47}$

\footnotetext{
4 Voir lIMA MARQUES Cláudia, "A proteção do consumidor: aspectos de direito privado regional e geral», in XXVII Curso de Derecho Internacional-OE A/CIJ, Ed. Secretaría General- Subsecretaria de Asuntos Jurídicos, Washington, 2001, p. 657-780, et republié, in El Derecbo Internacional Privado en Las Américas (1974-2000), Cursos de Denecho Internacional - vol. I (Parte 1), Editor Secretaria General-Subsecretaria de Asuntos Jurídicos, Washington, 2002, p. 1503-1622.
} 
Le thème de la nécessité et de l'opportunité de ces règles était aussi présent, dès le début de ce long parcours, dans les négociations préparatoires pour la CIDIP VII sur la protection des consommateurs. Effectivement, de 2001 à 2007, le grand thème de base était la nécessité et l'occasion pour le système interaméricain de défendre l'élaboration de normes spéciales pour la protection des consommateurs. Ce qui veut doncdire des normes différentes de celles de la CIDIP V de Mexico de 1994 (et plus protectrices de la personne), sur les thèmes procéduraux et administratifs de la protection des consommateurs, différents de ceux déjà traités par les CIDIPs, en particulier ceux de procédure civile internationale (telle que les petites créances, l'arbitrage électronique de consommation, forum privilégié, instruments de redress (réparation), etc.).

\section{Consensus sur l'occasion d'une CIDIP VII sur la protection des consommateurs}

Selon les mots déjà devenus classiques de Jean-Michel Arrighi, le consommateur est généralement le "protagoniste oubliés" ${ }^{48}$ des Traités internationaux, que ce soient les Traités interaméricains ou les CIDIPs, ou que ce soient ceux dédiés à l'intégration tels que le Traité de 1980 de l'ALADI et le Traité d'Assunción de 1991 du Mercosur, dans lesquels on ne rencontre jamais le terme «consommateur» ${ }^{49}$. De plus, dans aucune des CIDIPs jusqu'à ce jour signées le thème de la protection des consommateurs ne s'est mérité une attention spéciale (même pas une norme), alors que les thèmes furent concentrés sur le droit commercial international entre professionnels et sur les procédures civiles. Ce silence et cette exclusion de l'agenda peuvent avoir comme fondement la volonté d'éviter les conflits avec des lois nationales considérées comme d'ordre public international ${ }^{50}$, ou, peut-être encore, une certaine méconnaissance du sujet alors que les différences dans le domaine de la protection du consommateur ont toujours pesé à la faveur des pays plus industrialisés et exportateurs (il faut remarquer que parmi les 34 pays de l'OÉA, seuls le Canada et les ÉUA possèdent déjà des normes sur les conflits de lois, lesqueles bénéficient à lesquelles consommateurs). ${ }^{51}$

La vérité est que le thème de la protection des consommateurs (à l'exception du projet de LaHaye de 1980 sur l'achat et la vente de consommation élaborée par le Professeur étasunien Arthur von Mehren) ${ }^{52}$ jamais ne fut traité dans les Conventions. Ce thème ne fut pas non plus traité dans les Conventions classiques qui ont unifié les normes matérielles, ni

48 Arrighi, p. 126

49 Arrighi, p. 126

50 Tel que soutenu par: GARRO, Alejandro Miguel e ZUPPI, Alberto Luis. Comprazenta internacional de mercaderías, Ed. La Rocca, Buenos Aires, 1990 , p. 81 (traduction libre): «La raison principale d'exclure la vente aux consommateurs de la portée d'application est venue du fait de vouloir éviter un éventuel conflit entre les normes de la Convention et les lois d'ordre public de protection du consommateur. La législation spéciale de la protection des consommateurs a été incorporée durant les dernières années dans plusieurs systèmes juridiques, y compris dans plusieurs pays d'Amérique latine tel le Mexique.» Dans la même lignée, voir HARGAIN/MIHALI, Libre circulación..., p. 507.

51 HARGAIN/MIHALI, Libre circulación..., p. 506, citant l'opinion de Lible.

52 Toniollo, p. 96, commentant le projet de LaHaye. 
dans une loi-modèle d'UNIDROIT ou de la UNCITRAL ${ }^{53}$, et ne fut pas non plus l'objet d'une CIDIP ou d'une Convention de LaHaye en Droit international privé.

Seules les Résolutions de l'ONU ont eu une influence inspiratrice au sein des législations nationales dans les Amériques. Les législations de presque tous les pays de l'OÉA possèdent déjà des normes sur la protection des consommateurs ${ }^{54}$. On ne pourrait laisser sous silence les efforts de l'OCDE et ceux, plus réduits, du Code modèle de protection des consommateurs de la IUCO (Consumers International), du Code modèle de procédure civile pour l'Amérique ibérique et de l'actuel Code modèle ibéroaméricain sur les actions collectives.

Comme je l'avais constaté déjà en 2000, si tous les pays des Amériques connaissent la protection des consommateurs comme étant un ensemble de règles spéciales matérielles au niveau national (c'est-à-dire, comme des normes substantives de droit civil et administratif), ${ }^{55}$ seulement deux pays américains permettent une protection effective des consommateurs grâce à des règles spéciales de conflits de lois (ÉUA et Canada) ${ }^{56}$. Cette situation a commencé à changer en 2001 lorsque que le thème de la protection des consommateurs a commencé à faire partie de l'agenda des efforts de codification du Droit international privé à l’OÉA.

En d'autres mots, les trois pays qui firent des suggestions spécifiques pour la CIDIP VII sur la protection des consommateurs (Brésil, ÉUA et Canada) ainsi que les autres pays qui étaient présents à la réunion préparatoire de Porto Alegre sont effectivement d'accord avec le fait que la protection des consommateurs représente une lacune dans le système interaméricain actuel. Ces pays sont aussi en consensus sur l'opportunité que ceci représente pour le système interaméricain actuel de combler cette lacune, tel qu'il le fut montré clairement à la réunion de la CAJP/OÉA du 18 janvier 2007. En ce moment, les différents encore existants concernent la meilleure façon de remédier à cette lacune...

\section{La nécessité de la CIDIP VII sur la protection des consommateurs : définition des thèmes}

Si cette opportunité fait consensus absolu, les pays de l'OÉA voient cette «nécessité» de formes et de degrés différents, particulièrement en ce qui a trait aux thèmes. Je m'explique.

53 La loi-modèle de la UNCITRAL (qui, dans les faits, se consacre au commerce intemational), lorsqu'elle se penche sur le commerce électronique, indique expressément qu'il ne faut pas refouler les nomes tutélaires et semble vouloir exclure les contrats de consommation par le biais de l'exclusion de moyens informatiques de son champ d'application avec ce texte : "Art.1. Champ d'application - La présente loi* sera applicable à tous les types d'informations en forme de message de données utilisées dans le contexte d'activité commerciales. *La présente loi ne déroge à aucune norme juridique destinée à la protection des consommateurs.n

54 Voir l'étude du Ministère de la Justice brésilien, dénommée Atlas Geo-polititio de Defesa do Consumidor, sur son site www.mj.gov.br, dans la section du DPDC-SDE, qui remarque que seuls 4 pays peuvent être considérés comme ayant peu (ou pas) de règles spéciales pour la défense du consommateur en droit civil lesquels sont : Belize (Public Utilities Commission Acty Hire Purchase Act), Bolivie (Lay del Sistema de Regulación y Ley de Municipalidades, 1999), Guyana et la Republique Dominicaine. Voir dans la version publiée de l'Atlas, p. 18-19, en portugais et p. 140-141, en espagnol.

5 Voir le travail précédent du Ministére de la Justice brésilien dénommé Atlas Geo-politico de Defesa do Consumidor, sur son site www.mi govbr, dans la section du DPDC-SDE analysant toutes les lois avec comme bases les réponses des gouvernements: Atlas, p. 18-19, e p. 140-141, en espagnol.

56 Voir MASSE, Claude «Fondement historique de l'évolution du droit québecois de la consommation», in LAFOND, PierreClaude. Mélanger Claude Masse-En quête de justice et d'équité, Québec: Yvon Blais, 2003, p. $37-118$ et l'HEUREUX, Nicole. Droit de la Consommation. 4. ed., Québec: Yvon Blais, 1993. 
Le Canada a proposé une loi-modèle sur les contrats de consommation nationaux et internationaux, contenant six règles de juridiction spéciale pour les contrats de consommation internes (entre résidents de deux provinces différentes) et internationaux, défendant la compétence du juge du pays de la résidence habituelle du consommateur (Art. 2) et introduisant comme modèle le forum non conveniens pour les cas impliquant le consommateur (art. 5). Aussi, la loi-modèle canadienne considère nulle la clause d'élection du forum dans plusieurs cas, comme, par exemple si cette élection a été faite avant que le litige ne survienne ou encore si le choix fut fait dans le cadre d'un contrat d'adhésion (art. 6,1,a c/c art. 3,c). En novembre, le Canada a proposé complémentairement une norme unique sur la loi applicable (art. 7), avec une connexion subsidiaire à la résidence du consommateur (touriste et passif) et qui considère nulle la clause d'élection de la loi dans certains cas de consommation passive si cette lex contractus «a pour effet de priver le consommateur de la protection qui lui est garantie par la loi de sa résidence habituelle».

De l'analyse de ce projet on peut percevoir le consensus ainsi que deux conclusions importantes sur le thème ici discuté : a) le projet canadien fut rédigé de façon à démontrer qu'il reconnait l'existence d'une lacune dans la réglementation interaméricaine sur la protection des consommateurs. Ainsi, on peut voir un consensus sur la nécessité et sur l'opportunité d'élaborer des normes spéciales (et différentes de celles des CIDIPs précédentes) sur ce thème. Mais, b) on peut conclure que cette lacune existerait tant sur le thème de la juridiction que sur celui de la loi applicable, et, c) que la lacune serait large et existerait même dans le traitement des contrats de consommation nationaux ou internes des pays puisque le projet de loi-modèle canadien, de la façon dont il fut présenté le 30 novembre 2006, conclut que les pays de l'OÉA doivent changer leurs lois de DIP et de procédures civiles (nationales et internationales) afin de combler cette lacune générale, acceptant ainsi le modèle proposé comme celui étant le meilleur pour la région.

Effectivement, la loi-modèle proposée par le Canada est une «soft lan» (que les pays ne sont pas obligés de suivre, mais qui, en même temps, n'a pas à passer au double crible de l'Exécutif et du Législatif pour ratification, alors que les Conventions doivent y passer. Aussi, la soft law ne permet pas l'analyse de son inconstitutionnalité, au contraire des Conventions, lesquelles peuvent avoir une hiérarchie supérieure aux lois, mais qui ne pourront être ratifiées qu'après analyse de leur constitutionnalité). Mais, par les temps qui courent, les lois-modèles et les lignes directrices législatives détiennent un pouvoir symbolique très grand: celui de représenter le «meilleur» modèle législatif disponible, la «meilleure» solution, la «plus» effective ou intelligente, la «plus» consensuelle parmi celles existant dans la région.

Les ÉUA aussi ont présenté une loi-modèle sur les redress (réparation) pour les contrats nationaux et internationaux conclus avec les consommateurs, ensuite transformée en Guidelines et en trois autres lois-modèles sur des thèmes procéduraux (administratifs et pénaux) en défense des consommateurs. Mais toutes celles-ci ont des normes spéciales différentes de celles existant dans les autres CIDIPs, soit celles sur l'arbitrage international, sur la coopération judiciaire internationale et sur la reconnaissance des sentences. 
Des textes présentés par les ÉUA, pour leur originalité par rapport aux autres textes des CIDIPs concernant les relations entre deux commerçants, nous pourrions retirer un certain type de consensus émanant des ÉUA en ce qui a trait à la nécessité de lois spéciales de DIP pour la défense des consommateurs. On peut voir que, dans les interventions écrites des négociations, l'honorable délégation étasunienne recourt au texte de la CIDIP V de 1994 en indiquant que cette Convention, pas ratifiée par les ÉUA, pourrait être suffisante et même utile pour la défense des consommateurs en matière de conflits de lois. Effectivement, dans son intervention officielle sur la proposition brésilienne durant les négociations subséquentes, les ÉUA citèrent plusieurs fois les normes de la CIDIP V sur les contrats internationaux pour faire remarquer que celles-ci pourraient être utilisées aussi en matière de protection des consommateurs (particulièrement l'art. 11 sur les normes impératives du forum) et que la formulation de la CIDIP VII devrait se rapprocher de celle de la CIDIP V.

Ainsi, il est possible de conclure que, selon la position étasunienne actuelle, il y a la nécessité et l'opportunité au sein du système interaméricain d'élaborer des normes spéciales de protection des consommateurs en particulier sur les thèmes de procédure civile (même s'il existe des CIDIPs sur ces mêmes thèmes pour les contrats entre fournisseurs ou commerçants, comme celle sur l'arbitrage), mais par le biais de lois-modèles nationales et internationales. Dans ce sens, les ÉUA reconnaissent les spécificités et la nécessité d'une protection spéciale pour le consommateur dans sa relation avec les fournisseurs de produits et de services. Par contre, en matière de loi applicable, il reste des doutes à propos de la CIDIP V, laquelle n'est pas en vigueur aux ÉUA, à savoir si celle-ci serait suffisante ou positive pour la protection des consommateurs de la région.

Donc, de l'analyse de ces projets présentés par les ÉUA et par ses interventions jusqu'à maintenant, il est aussi possible d'apercevoir un consensus ainsi que deux conclusions importantes sur le thème ici discuté : a) le consensus porte sur l'existence d'une lacune dans la réglementation interaméricaine sur la protection des consommateurs, aussitôt donc, il y a consensus sur la nécessité et sur l'opportunité d'élaborer des normes spéciales (et différentes de certaines des CIDIPs existantes) sur le thème, particulièrement par le biais de loismodèles et de soft laws ayant effet sur les contrats nationaux et internationaux de consommation; b) cette lacune n'existerait pas ou du moins, ne serait pas aussi importante, sur le thème exclus de la juridiction et qu'il y a des doutes sur son existence au sujet du thème de la loi applicable face à la CIDIP V de 1994, e, c) que cette lacune existerait même dans le traitement des contrats nationaux de consommation car la loi-modèle sur l'arbitrage de consommation (électronique) restreint son application aux contrats internationaux de consommation, s'appelant Model Law for Electronic Arbitration of Cross Border Claims. Ces lois modèles serviraient donc les pays de l'OÉA pour les aider à modifier leurs normes procédurales civiles, celles sur les actions individuelles, collectives et de petites créances (nationales et internationales) et celles sur l'arbitrage international, afin de combler cette lacune générale, acceptant ces différents modèles proposés comme les meilleurs de la région. 
Le Brésil aussi considère que cette lacune existe, au point qu'il a proposé une Convention pour la CIDIP VII sur la protection des consommateurs. Mais il considère cette lacune comme étant plus grave et nécessitant une action immédiate de l'OÉA simplement en matière de détermination de la loi applicable aux contrats entre les fournisseurs de produits et services et les consommateurs et ce, pour trois raisons que je résumerai ainsi :

1) La CIDIP $\mathrm{V}$ de Mexico de 1994, avec son autonomie des parties pour élire n'importe quelle loi du monde même celle d'un État non partie (Art. 2) et sans aucune limite imposée par son texte (Art. 7,1) ${ }^{57}$ au-delà du très timide article 11 sur l'application des normes impératives (lequel, dans la pratique, laisserait le consommateur touriste sans la protection des normes impératives de son pays de domicile tout en laissant à la discrétion du juge du «forum» l'usage des règles impératives étrangères si le consommateur a contracté à distance via le commerce électronique sans sortir de son pays) ${ }^{58}$, est considérée comme étant très large même pour des relations entre deux sujets égaux, les fournisseurs. Si large, en fait, qu'elle ne fut pas ratifiée par le Brésil et est considérée comme étant très peu effective pour la protection des consommateurs. Cette CIDIP V de Mexico fut conçue pour des contrats entre sujets égaux, des professionnels pourvus de départements juridiques, des entreprises et des personnes juridiques déjà habituées à agir dans le marché interaméricain, dotés de compétences linguistiques et d'un pouvoir de marchander en plusieurs aspects différent et supérieur à ceux des consommateurs, des personnes physiques, profanes, ayant des carences linguistiques et juridiques beaucoup plus importantes. Ces consommateurs personnes physiques, avec la croissance du commerce électronique et du tourisme de masse, participent aujourd'hui de façon plus importante au marché régional. Mais cette participation se fait encore sans grande confiance et sans protection spécifique, ce qui amène les magistrats à appliquer toutes les normes nationales de protection du consommateur comme si elles étaient toutes des normes d'ordre public international, des lois et pouvoirs de police ou des normes impératives et d'application immédiate ${ }^{59}$. Afin de changer cette situation actuelle dans laquelle les clauses d'élection du forum et d'élection de la loi dans les contrats d'adhésion de consommation sont considérées abusives et nulles, par la loi interne considérée d'application impérative, il est nécessaire que soient crées des normes spéciales et spécifiques sur les conflits de lois pour les contrats internationaux de consommation, assurant ainsi une véritable prévisibilité sur la validité de la clause d'élection de la loi et une véridique assurance pour les fournisseurs et les consommateurs.

\footnotetext{
${ }^{57}$ NOODT TAQUELA, Marái Blanca, Chap. 25, in FERNÁNDEZ ARROYO, Diego (Org). Derecho Internacional Prizado de los Estados del Mercosur-Angentina, Brasil, Paraguay, Uruguay, Ed Zavaria, Buenos Aires, 2003, p. 996-1000.

${ }^{58}$ Tel que vu dans mon cours à l'OÉA en 2002, p.697 et s

${ }^{59}$ Tel que vu dans mon cours à l'OÉA en 2002, p.697 et s
} 
2) Dans son premier article, la CIDIP V de Mexico de 1994 détermine de façon expresse que la Convention s'applique à tous les contrats à part ceux déterminés exclus de son champ d'application par déclaration de la part de chaque pays au moment de sa ratification, comme, par exemple, les contrats de consommation. Cette règle trouve son origine dans la proposition initiale du Professeur Siqueiros (Mexique), qui exclue les contrats de consommation de façon expresse du champ d'application de la CIDIP V. Mais, dans les négociations, la question a de savoir si cette CIDIP s'applique ou non à ces contrats resta ouverte, les États pouvant, dans leurs relations bilatérales avec les autres, décider s'ils feraient ou non cette déclaration. Le Mexique et le Venezuela, les seuls pays où la CIDIP V est en vigueur, ne semblent pas avoir fait une telle déclaration, ce qui pourrait peut-être expliquer pourquoi l'honorable délégué mexicain présent à la réunion de Porto Alegre suggéra, en en soulignant limportance, d'inclure les petites entreprises et les personnes juridiques de petite portée en tant que consommateurs lorsque ceux-ci agissent en position de vulnérabilité plus grande de façon à limiter l'autonomie des parties et protéger ces «petites» personnes juridiques au sein du marché interaméricain, comme le fait la loi mexicaine actuelle dans les relations nationales. Le Mexique suggéra donc que ce type de consommateurs puisse être inclus dans la définition présente dans la proposition brésilienne pour la CIDIP VII.

On peut noter que le Brésil aussi n'a pas ratifié la CIDIP V, mais la position officielle brésilienne actuelle à cet égard est que si un jour elle le fait, elle déclarerait que cette CIDIP ne s'applique pas aux contrats entre consommateurs et fournisseurs de produits et services. Deux problèmes d'insécurité et de prévisibilité apparaissent ainsi à l'horizon. Premièrement, dans les relations avec les consommateurs brésiliens, la CIDIP $V$ ne pourrait être évoquée, même si l'autre pays en est partie et n'a pas fait la déclaration, par exemple, dans un futur hypothétique, les ÉUA. Deuxièmement, l'insécurité est plus grande puisque dans l'absence d'une définition conventionnelle du consommateur personne physique, telle celle présente dans les trois propositions à l'examen en ce moment, le Brésil utilisera la définition (de sa lex for) du Code de Défense du Consommateur brésilien (Loi 8.078/90), laquelle est très large, incluant toutes les personnes physiques ou juridiques qui agissent en tant que destinataires finaux d'un produit ou d'un service (art. 2 de la loi 8.078/90), réduisant ainsi le champ d'application de la CIDIP V de 1994, dans le cas où elle serait ratifiée. De cette façon, l'entérinement de la proposition brésilienne de la CIDIP VII, avec une définition plus restrictive du consommateur, aurait ainsi pour effet de faciliter la ratification de la CIDIP V par le Brésil.

Le mérite de la proposition brésilienne est de complémenter le système interaméricain, promouvant en même temps la ratification d'autres Conventions actuelles (autres CIDIPs comme celle sur le domicile, les preuves et informations de droit étranger, la CIDIP V sur les contrats internationaux, etc.) et futures (comme par exemple la proposition uruguayenne sur la juridiction et/ou d'autres futures loismodèles) puisque le projet brésilien vise à réguler simplement la loi applicable aux contrats internationaux de consommation et non pas les contrats nationaux entre fournisseurs et consommateurs. La proposition brésilienne apporte une définition plus restreinte du consommateur (personne physique), mais protège le consommateur 
touriste (consommateur mobile, qui sort de son pays de domicile pour contracter) et le consommateur passif (ou non mobile) et permet l'autonomie des parties de façon limitée, validant ainsi la clause d'élection de la loi même dans les relations impliquant les consommateurs. De cette façon, elle ouvre la porte pour la ratification de la CIDIP V de Mexico puisque, si l'élection de la loi est possible même dans les contrats de consommation, il n'y aurait pas de raison pour que celle-ci ne soit pas possible pour les contrats entre deux sujets égaux et professionnels. De plus, la proposition brésilienne pour la CIDIP VII apporte une série d'assurances afin d'augmenter la confiance des consommateurs envers le marché régional. Ainsi, lorsque le consommateur est passif, elle impose un test de la loi la plus favorable au consommateur, de façon à éviter la discrimination envers les consommateurs et l'usage de standards légaux différents, conformes à ceux du domicile du consommateur, échappant ainsi à l'élection de lois facilitatrices et contraires aux droits du consommateur. L'objectif est d'amener une véritable prévisibilité et une assurance pour l'une et l'autre des parties au contrat de consommation, incluant les contrats d'adhésion, puisque dans la majorité des cas, la loi prévue dans ce dernier type de contrat (et validée expressément par la liste de lois parmi lesquelles le choix peut être fait) régulera les relations entre consommateurs et fournisseurs. En résumé, elle réglementera donc l'accomplissement volontaire de ce contrat, sans la nécessité de recourir à la Justice ou à un forum d'arbitrage pour ce faire. La proposition brésilienne promeut ainsi l'élection de la loi dans le contrat ainsi que le devoir de transmettre cette information préalablement au consommateur, afin que ce dernier puisse choisir une meilleure protection du consommateur en cas de conflits créant des éléments de connexion spéciaux.

3) Suivant l'exemple des pays de l'Union Européenne, le Brésil considère qu'il est meilleur et plus efficace de réguler le thème de la loi applicable aux contrats internationaux et le thème de la juridiction spéciale pour le consommateur dans ces contrats (et délits) par le biais d'instruments différents et de logique indépendante (définitions différentes, connexions différentes, puisque le droit fondamental d'accès à la justice est différent du droit de protection des intérêts économiques du consommateur, etc.), qu'elles soient des lois nationales (modèles) ou internationales (Conventions), promouvant ainsi la signature de Conventions et instruments sur chacun de ces thèmes.

À ce sujet, c'est pourquoi l'opinion brésilienne croit qu'il est aujourd'hui opportun de combler la lacune la plus grave en matière de loi applicable aux contrats internationaux (plutôt qu'en matière de juridiction puisque le Brésil possède déjà un forum spécial pour les consommateurs, utilisé avec succès dans des cas internationaux et invalidant les clauses d'élection du forum dans les contrats d'adhésion). La doctrine brésilienne a suivi l'échec de la Conférence de LaHaye, qui tenta sans succès pendant dix ans de créer une norme générale sur la juridiction et de valider l'élection du forum dans les contrats de consommation. De plus, elle reconnait les efforts contenus dans la proposition uruguayenne d'une Convention de base pour la région. Les thèmes de procédure, de juridiction, de validité de la clause d'élection du forum et de l'arbitrage de consommation ont des liens très étroits avec les droits humains et fondamentaux d'accès à la justice, tout comme, dans la vision latino-américaine, avec 
les normes d'ordre public et ainsi méritent des études spéciales. Dans ce sens, et aussi pour cause de sa complexité actuelle et de l'inexistence d'un consensus à son sujet et ce, même à l'interne, le thème de la juridiction fut exclu de la proposition brésilienne. Dans les processus d'intégration économique dans la région le même phénomène put être observé alors que même le Mercosur ${ }^{60}$ ne réussit pas à faire entrer en vigueur les normes de juridiction spéciales pour la protection des consommateurs prévues par le Protocole de Santa Maria. ${ }^{61}$

Les grandes Conventions (Hard Law) et même les lois-modèles (Soft Law) sur les thèmes des contrats et des procédures civiles internationales régulent généralement simplement le commerce international entre deux professionnels (B2B) et excluent de leur champ d'application les consommateurs, que ce soient celles se référant à l'achat et la vente ${ }^{62}$ ou encore celles se référant au commerce électronique ${ }^{63}$ et même à l'arbitrage $^{64}$. Il est possible de rencontrer des Conventions fortes et justes en matière de délits ou d'accidents de consommation, comme la Convention de LaHaye de 1973 sur la responsabilité extracontractuelle ou pour les produits complexes ou industrialisés. L'opinion brésilienne est qu'il n'existe pas de lacune en matière de responsabilité pour les produits défectueux.

Pour conclure, il existe clairement un consensus brésilien sur la nécessité de normes spéciales dans le système interaméricain en matière de conflits des lois et en matière de contrats internationaux de consommation, mais on y reconnait l'importance

6. Voir SZAFIR, Dora El consumidor en el derecho comunitario- Proyeto de protocolo de defensa del consumidor del Mercosur, FCU, Montevideo, 1998, p. 8 et s.

61 Au sujet des problèmes de ce Protocole et des tentatives du Mercosul, voir: ARROYO, Diego P. Femández. "La nueva configuración del Derecho Internacional Privado del MERCOSUR: ocho respuestas contra la incertidumbres. In Rerista de Direito do Mercosul, a. 3, n. 4, p. 38-53, ago. 1999, p. 41 et ARROYO, Diego P. Fernández. Denecho Internacional Prizado de los Estados delMERCOSUR - Argentina, Brasil, Unuguay e Panaguay. Buenos Aires: Zavalia, 2003, p.1032; MARQUES, Cláudia Lima «Direitos do consumidor no Metcosul: algumas sugestões frente ao impasse». RDC, n. 32, p. 16-44, out./dez. 1999, p. 34-35

Q Voir les exclusions de la Convention de LaHaye sur la loi applicable aux contrats internationnaux d'achat et de vente de 1986 (Art.2. The Convention does not apply to ....) sales of goods bought for personal, family or bousehold use; it does, bonever, apply if the seller at the time of the conciusion of the contract nieither knew nor ought to have knon:n that the goods were bought for any such use. Art. 5 . The Convention does not determine the law applicable to ... d) the effects of the sale in respect of any person other than the parties.), dans la "Convention» UNCITRAL de 1980 sur l'achat et la vente internationale de marchandises - Vienne» (Art. 2. La présente convention ne s'appliquera pas aux achats et ventes: a) de marchandises achetées pour usage personnel, familier ou domestique, sauf que le vendeur à tout moment avant la célébration du contrat ou au moment même de sa célébration, ne sera pas tenu ni ne devra avoir pris connaissance du faire que la marchandise en question est achetée pour un tel usage;...).

Q3 Voir l'exclusion dans la nouvelle Convention de l'ONU de 2005, United Nations Convention on the Use of Electronic Communication in International Contracts -2005 (Article 2. Exclusions 1. This Convention does not apply to electronic communications relating to any of the following: a) Contracts conclude for personal, family or household purposes;) et dans la plus connue loi-modèle de l'UNCITRAL (Loi Modèle de l'UNCITRAL sur le commerce électronique-UNCITRAL-1996. (Art. 1. Champ d'application. La présente loi* est applicable à tous les types d'information en forme de messages de données utilisés dans le contexte d'activités commerciales * La présente loi ne déroge à aucune norme juridique destinée à la protection des consommateurs.)

64 Voir ces exclusions dans la Convention de New York de 1958 (ONU), la Convention de Washington de 1965 (ONU), la Convention de Panama de 1975 (OÉA) et la Convention interaméricaine sur l'application extraterritoriale des sentences d'arbitrage étrangères de 1979 (OÉA) et l'exclusion de la loi-modèle sur l'arbitrage commercial de la UNCITRAL (Ant. 1. Scope of application. (1) This Lay applies to international commercial arbitration (relationship of a commercial nature)... (5) This Las' shall not affect any other law of this State by irtue of wich certain disputes may not be submitted to arbitration or may be submitted to arbitration only according to protisions other than those of this Lai:) 
des problèmes de procédure civile, lesquels découlent surtout de l'opportunité et de la consolidation de la proposition présentée.

\section{B) La double-leçon de la réunion préparatoire de Porto Alegre : une Convention en matière de loi applicable en tant que consensus latino-américain et la possibilité de l'approbation de lois-modèles et de Conventions lors de la conférence finale pour la CIDIP VII}

Sans vouloir faire une conclusion stricto sensu de cet article déjà très long, j'aimerais profiter de la prochaine section pour faire quelques observations finales sur les leçons que l'on peut tirer de la réunion de Porto Alegre et des négociations subséquentes.

\section{Consensus latino-américain sur la nécessité et sur l'opportunité d'une Convention en matière de loi applicable : l’appui à la proposition brésilienne}

L'impressionnant consensus latino-américain sur la nécessité et sur l'opportunité d'adopter une Convention en matière de loi applicable pour la CIDIP VII, atteint lors de la réunion préparatoire, reste une des grande leçon à tirer de cette dernière. Ce consensus fut possible grâce au conductorat de l'Argentine et se manifesta en un appui à la proposition brésilienne, aujourd'hui fortement améliorée par les suggestions des experts et des délégués.

Si on considère que, au contraire des ÉUA et du Canada, les règles de Droit international privé des autres pays de l'OÉA et plus particulièrement celles des pays latino-américains de tradition civiliste ne traitent pas la protection des consommateurs d'une forme spéciale et que presque tous ces pays ont déjà - plutôt - des normes nationales et substantives qui protègent suffisamment les consommateurs, il ne faut pas s'étonner que ces pays latinoaméricains aient appuyé l'élaboration d'une Convention sur la loi applicable aux contrats de consommation, comme la proposition brésilienne.

La réunion de Porto Alegre a ainsi démontré l'existence d'un consensus parmi les pays latino-américains sur la nécessité de mener à terme les efforts afin que la CIDIP VII se conclue par une Convention sur la loi applicable. Ce consensus découle de l'obligation spéciale de ces pays face à la lacune qui existe aujourd'hui. Comme les Conventions - au contraire des lois-modèles qui présupposent un consensus au sujet du meilleur et du plus efficace modèle législatif existant - lient seulement les pays les ratifiant, la réunion de Porto Alegre permit de constater que toutes les délégations présentes sont disposées à négocier une Convention sur le sujet afin de répondre aux nécessités législatives de ces pays latino-américains, lesquels sont déjà habitués à utiliser la voie de la Convention sur les thèmes qui font consensus.

S'il y a une lacune législative dans les règles de DIP dans les pays d'Amérique latine sur le thème de la protection des consommateurs, la doctrine - au contraire - depuis les années quatre-vingt du vingtième siècle, est très consistante et réclame des normes spéciales de DIP de protection des consommateurs dans les contrats internationaux, non seulement 
au Brésil et en Argentine, mais aussi et initialement au Venezuela ${ }^{65}$, en Uruguay ${ }^{66}$, au Paraguay ${ }^{67}$, et Pérou ${ }^{68}$ au en Bolivie ${ }^{69}$.

La réunion de Porto Alegre démontra qu'il fait consensus parmi tous les pays latinoaméricains que l'OÉA offrirait une excellente contribution si elle élaborait des normes spéciales sur l'indication de la loi applicable aux contrats internationaux de consommation, thème classique du DIP. Ici il y a consensus au sujet de sa nécessité et de son opportunité. Sur le thème de la juridiction de consommation, il parait avoir moins d'unanimité pour le moment, à cause de sa complexité et de l'expérience récente de l'échec à LaHaye, mais le projet uruguayen est considéré comme important pour initier les premiers pas dans ce sens. Aussi, lors de la réunion de Porto Alegre, on a établi un consensus parmi tous les pays latino-américains présents sur le fait que ces deux thèmes (droit applicable et juridiction) considérés comme des droits fondamentaux dans plusieurs pays devraient être traités par Convention, justement à cause de son importance et de sa relation directe avec les valeurs et les normes constitutionnelles.

À mes yeux, ce consensus ne fut pas atteint seulement parce que le protagoniste de cette proposition pour la CIDIP VII est le Brésil, un pays latino-américain. Au sein du Forum virtuel, les excellentes suggestions des délégations et des experts d'Argentine, d'El Salvador, d'Uruguay, du Paraguay, du Mexique, entre autres, ont démontré qu'il s'agissait ici d'un consensus doctrinaire à la base. Et, à l'origine des idées suivies par la proposition brésilienne, on retrouve des doctrines souvent citées dans ces pays ${ }^{70}$ comme, par exemple, sur la protection du consommateur touriste par le biais de l'autonomie limitée des parties et de la loi la plus favorable au consommateur qui est argentine ${ }^{71}$, en plus des influences communes de la doctrine continentale européenne, particulièrement celle d'Erik Jayme et d'autres juristes allemands, français, italiens, portugais, espagnols et hollandais.

(5) Voir HERNÁNDEZ-BRETON, Eugenio «Las obligaciones convencionales en la Ley venezolana de Derecho internacional privadon, in DECITA (Buenos Aires), 01.2004, p. 278 et s. et son oeuv re plus classique sur la clause d'élection du forum dans les contrats intemationaux d'adhésion HERNÁNDEZ-BRETON., Eugenio. Internationale Gerichtsstandsklauseln, in Allegemeinen Geschäftsbedingungen, Frankfurt am Main: Peter Lang, 1993, et plus récemment, HERNÁNDEZ-BRETON., Eugenio. Problemas Contemporáneos del Derecbo Procesal Ciril internacional Venezolano. Caracas: Ed. Sherwood, 2004

66 Voir d'Uruguay: ARRIGHI Jean Michel, «La Proteccion de los Consumidores y el MERCOSUR», Rerista Direito do Consumidor, São Paulo, v. 2 (1992), p. 126 et s., ORDOQUI CASTILLA, Gustavo Derecbo del Consumo, Ed. Del Foro, Montevideo, 2000, p 5-7, SZAFIR, Dora Consumidores, FCU, Montevideo, 2000, p. 17 et s., SZAFIR, Dora: El consumidor en el derrecho comunitario-Proyeto de protocolo de defensa del consumidor del Mencosur, FCU, Montevideo, 1998; HARGAIN, Daniel e MIHALI, Gabriel, Cinculación de Bienes en el Mercasur, Ed. Júlio César Faira, Montevidéo, 1998, HARGAIN, Daniel e MIHALI, Gabriel. Contratación Mercantil internacional en el Merosur Ed. Júlio César Faira, Montevideo, 1993, p. 113 et s. et TELLECHEA BERGMAN, Eduardo, La dimensión judicial del caso prizado internacional en el ámbito regional, Montevidéo: FCU, 2002, DELPIAZZO, Carlos E. e VEIGA, Marí José. Lecciones ae Derecho Telemático. Montevideo, FCU, 2004.

G Voir MORENO RODRÍGUEZ, José Antonio. Teoria da Causa, Asunción: Ed. Intercontinental, 1996, p. 384 et s.

\& Voir VEGA MERE, Yuri. Contratos de consumo. Lima: Grijley, 2001, p. 171 et s

( Voir l'article de l'exeperte bolivienne, republié au Brésil TRINAJEROS ARCE, Érika Patrícia. «La protección del consumidor electrónico en los países del MERCOSUR», in Reizsta de Direito do Consumidor, vol. 54 (2005), 173-192.

70 Voir LIMA MARQUES, Cláudia, Confiança no comércio eletrónico e a protegãa do consumidor, São Paulo: Editora Revista dos Tribunais, 2004, p. 301 ets

71 Les influences principales furent : BOGGIANO, Antonio, «International Standard Contracts», in Recueil des Cours, 1981, I, t. 170, Nijhoff, Dordrecht, 1982, p. 138 et s., "The Contribution of the Hague Conference to the Development of Private International Law in Latin America. Universality and genius locis, Recueil des Cours, 1992, II, t. 233, Nijhoff, Dordrecht, 1993, p. 99-266 et Curso de Dericho Internacional Privado, Abeledo-Perrot, 2000, p. 280; TONIOLLO Javier Alberto, «La protección internacional del 
Depuis la décennie des années 90 du vingtième siècle, la doctrine civiliste et du DIP des pays d'Amérique latine revendique une plus grande protection pour le consommateur dans ses relations et transactions, particulièrement en Argentine ${ }^{72}$ et au Brésil. ${ }^{73}$

Notons que ce fut le Professeur argentin Boggiano ${ }^{74}$, en observant le fonctionnement de la Convention de Rome de 1980 et les règles ouvertes de la doctrine étasunienne (the better law), qui proposa, pour la protection des consommateurs, une règle d'autonomie limitée des parties et inversa le favor offerentis pour le favor consumidor dans les contrats d'adhésion : l'élection des parties prévaudra seulement si le choix ainsi fait est la meilleure loi, la loi la plus favorable au consommateur, et ainsi devra, advenant le cas contraire, appliquer la loi du domicile du consommateur. Les normes d'ordre public international et les normes de police ou impératives prévaudraient ainsi en tant que limites générales à l'autonomie des parties. ${ }^{75}$

consumidor-Reflexiones desde la perspectiva del Derecho Internacional Privado Argentino», Rerista de Derecho del Merosur, $\mathrm{v}$. 2, n. 6, dic. 1998, p. 96, DREYZIN DE KLOR, Adriana, El Mercosur-Generador de una nueva fuente de derecho intemacional privado, Ed. Zavalia, Buenos Aires, 1997 e STIGLITZ, Gabriel, El derecho del consumidor en Argentina y en el Mercosur, in Derecho del Consumidor, Buenos Aires, vol. 6, 1995, p. 20 et s. et LORENZETTI, Ricardo Luis. Tratado de los contratos. Buenos Aires: Rubinzal-Culzoni, 1999-2000, vol. I, II e III.

72 Voir, pour l'Argentine, BOGGIANO, Antonio, «International Standard Contracts», in Recueil des Cours, 1981, I, t. 170, Nijhoff, Dordrecht, 1982, p. 138 et s., BOGGIANO, Curso de Derecho Internacional Privado, Abeledo-Perrot, 2000, p. 280; TONIOLLO Javier Alberto, «La protección intemacional del consumidor-Reflexiones desde la perspectiva del Derecho Intemacional Privado Argentino", in Retista de Derecho del Mercosur, v. 2, n. 6, dic. 1998, p. 96, FERNÁNDEZ ARROYO, Diego (Org.). Derecho Internacional Privado de los Estados del Mercosur- Argentina,Brasil, Paraguay, Uruguay, Ed. Zavaria, Buenos Aires, 2003, DREYZIN DE KLOR, Adriana, El Mercosur- Generador de una nueva fuente de derecho internacional privado, Ed. Zavalia, Buenos Aires, 1997 e STIGLITZ, Gabriel, «El derecho del consumidor en Argentina y en el MERCOSUR», in Derecho del Consumidor, Buenos Aires, vol. 6, 1995, p. 20 et s. et LORENZETTI, Ricardo Luis. Comercio electrónico. Buenos Aires: Abeledo-Perrot, 2001, p. 256 et s., ainsi que dans notre livre conjoint, LORENZETTI, Ricardo et LIMA MARQUES, Claudia. Contratos de Servicios a los consumidores, Ed. Rubinzal-Culzoni, Buenos Aires/Santa Fé, Argentina, 2005, GHERSI, Carlos Alberto (Director), MercosurPerspectivas desde el derecho privado- Segunda Parte, Editorial Universidad, Buenos Aires, 1996, DREYZIN DE KLOR, Adriana, "Derecho Aplicable al comercio electrónico", in El Futuro de la codificación del Derecho internacional prizado en América- De la CIDIP VI a la CIDIP VIII, Org. Diego Fernández Arroyo et Fabio Mastrángelo, Córdoba: Ed. Alveroni, 2005, p. 104 et s. et FELDESTEIN DE CÁRDENAS, Sara Lidia «Arbitraje electrónico: una solución para y por Intemet), in DeCITA (Buenos Aires), vol. 02.2004,p.275 et s. et ALL, Paula María «El régimen general de los contratos intemacionales en el ámbito del Mercosur. Una asignatura pendienten, in Derecho Internacionaly de la Integración. Nr. 03 (2006), Santa Fé, p. 171 et s.

73 Pour le Brésil, voir la position de la doctrine in LIMA MARQUES, Cláudia, Confiança no comércio eletrônico e a proteção do consumidor, São Paulo: Editora Revista dos Tribunais, 2004, p. 371 et s., LIMA MARQUES, Claudia (Org.), Estudos sobre a proteção no Brasil e no Mercosul, Editora Livraria dos Advogados, Porto Alegre, 1994, LIMA MARQUES, Claudia. «Some recent developments in Private International Law in Brazily, Japanese Yearbook of Priate International Lan, vol. 4, 2002, Tokio, p. 13-50, et TEPEDINO, Gustavo, Multipropriedade Imobiliária, Saraiva, São Paulo, 1993, DE LUCCA, Newton. O «Mercosul e a defesa dos consumidores brasileirosm, in Retista de Derecho del Merossur, Buenos Aires, ano 2, n. 5, octubre de 1998, BATISTI, Leonir. Direito do Consumidor para o Mercosul, Ed. Juruá, Curitiba, 1998 RIZZATTO NUNES, Luiz Antônio, Comentários ao CDC, Saraiva, São Paulo, 2000,p. 349 ets., KLAUSNER, Eduardo. «Reflexões sobre a proteção do consumidor brasileiro nas relações intemacionais de consumom, in TIBURCIO, Carmen e BARROSO, Luís Roberto. O Dineito Internacional Contemponáneo, Estudo em Homenagem ao Professar Jacob Dolinger, Renovar: Rio de Janeiro, 2006, p. 375-420 e ARAÚJO, Nádia de. «Contratos internacionais e consumidores nas Américas: análise da proposta brasileira para uma Convenção interamericana na CIDIP VII», in Revista Brasileira de Direito Internacional, Curitiba, ano I, nr. 02, 2005, p. 4-38, et ARAÚJO, Nádia de Contratos internacionais e consumidores nas Américas e no Mercosul: análise da proposta brasileira para uma Convenção interamericana na CIDIP VII, in PIMENTEL, Luis Otávio, ESPLUGUESMOTA, Carlos et BARRAL, Welber ( $\mathrm{Org}$.). Direito Internacional Privado- União Européia e Mercosul. Flotianópolis: Fund. Boiteux, 2007,p.129 et s. et ARAÚJO, Nádia de. "Contratos internacionais de consumo nas Américas e no Mercosul: regulamentação atual e proposta brasileira para uma Convenção interamericana na CIDIP VII», in TIBURCIO, Carmen et BARROSO, Luís Roberto. O Direito Internacional Contemporânes, Estudo em Homenagem ao Professor Jacob Dolinger, Renovar: Rio de Janeiro, 2006, p. 705-736, et RICHTER, Karina. Consumidor e Mercosul. Curtiba: Juruá, 2003, FELLOUS, Beyla Esther. Proteção do consumidor no Mercosul e na União Européia, São Paulo: RT, 2003 et GUERREIRO CRUZ, Carolina Dias. Contratos Intemacionais de Consumo - Lei aplicável. Rio de Janeiro: Forense, 2006

74 Voir, Boggiano, dans son texte The Contribuition, p. 138 e 139.

75 Boggiano, The Contribuition, p. 137 
Ces idées furent incorporées dans la proposition originale de 2000, qui sera ensuite connue en tant que «Proposition Lima Marques » ${ }^{76}$, laquelle fut publiée aux ÉUA, au Brésil et en Argentine pour ensuite être présentée et débattue lors de conférences aux ÉUA, au Canada, en Argentine, en Uruguay et en Europe (Allemagne, France, Grande-Bretagne). Mais le point fort de l'acceptation de cette proposition est l'inclusion du thème de la défense des consommateurs dans la «Déclaration de professeurs sud-américains de Droit international privé à propos du futur du processus de la CIDIP» de décembre 2003, connue sous le nom de Déclaration de Cordoba.

Ainsi, les professeurs alors réunis à l'université de Cordoba ont reconnu cette lacune au sein de notre système interaméricain de Conventions, lequel s'est penché sur plusieurs thèmes, mais jamais n'a régulé de façon spéciale le commerce interaméricain entre les consommateurs et les fournisseurs de produits et service, commerce en constante croissance dans la région.

Nous avons peu d'espoir pour l'instant envers la CIDIP V. Celle-ci fut ratifiée seulement par le Mexique et le Venezuela et, depuis 1998, alors que le fameux professeur étasunien Friedrich $K$. Juenger dans une lettre adressée au Département d'État interrogea les motifs de sa non-acceptation, on lui répondit que la version anglaise de la Convention contiendrait plusieurs «défauts» de façon à rendre inviable son acceptation par les ÉUA. En 2001, le Canada aussi adopta cette position et abonda dans le même sens au sujet de ses difficultés à ratifier la CIDIP V de 1994 sur les contrats intemationaux dans sa version anglaise et française ${ }^{77}$. Si le Canada et les ÉUA n'ont pas ratifié la CIDIP V, il y a peu de chances que cette Convention permette la réalisation du dialogue nord-sud, qu'elle facilite la croissance du commerce au sein des Amériques, ou encore moins qu'elle y implique les consommateurs.

Il me semble que les efforts de traiter conjointement les thèmes du contrat intemational entre deux professionnels (B2B) et du contrat entre consommateurs et fournisseurs (B2C) sont voués à l'echec ${ }^{78}$, de là la sagesse de l'agenda établi par l'OÉA pour la CIDIP VII.

À mon avis personnel, il est nécessaire de créer chez les consommateurs une plus grande confiance envers le système interaméricain et ainsi permettre qu'un plus grand nombre de contrats de consommation soient conclus dans la région. L'élaboration d'une CIDIP VII contenant des règles spéciales de protection des consommateurs est une chance en or afin de réaliser ce dialogue et une nouvelle occasion pour la CIDIP V, maintenant que se distinguent clairement les normes des CIDIPs pour les contrats commerciaux internationaux et les contrats internationaux de consommation.

76 Cette dénomination trouve son origine dans le texte du délégué argentin pour l'OÉA, Diego Femández Arroyo, qui analysait la «propuesta Lima Marques», et qui fut reproduit dans le livre El Futuro de la codificación del Derecho internacional privado en AméricaDe la CIDID VI a la CIDIP VIII, Org. Diego Fernández Arroyo e Fabio Mastrángelo, Córdoba: Ed. Alveroni, 2005, p. 74.

$\pi$ Voir le rapport du grand professeur venezuelien, PARRA-ARANGUREN, Gonzalo. «La sexta conferencia especializada interamericana sobre derecho internacional privado (CIDIP -VI, Washington, 2002)", in Rerista de Derecho (Tribunal Supremo de Justicia, Caracas, Venezuela), vol 6, p. 204-205

78 Ce qui pourrait donner un autre exemple d'échec au sein de l'OÉA. En 2002, la Résolution de la CIDIP VI (CIDIP-VI/Res.6/ 02) indique qu’on ne peut réussir à atteindre um consensus sur le projet étasunien de «règles Uniformes Interaméricaines en matière de Documents et firmes électroniques», et le fait que celui-ci ne différenciait pas suffisamment les contrats de consommation des autres types de documents, peut être à l'origine de cet échec. 


\section{L'agenda ouvert et double de la CIDIP VII sur la protection des consommateurs : sagesse de la pluralité permettant le dialogue des différences.}

Après ce long rapport, l'observateur aguerri pourrait se demander pourquoi l'agenda de la CIDIP VII continua ainsi, ouvert et double. En d'autres mots, on peut s'interroger sur pourquoi la réunion de Porto Alegre n'a pas permis de décider définitivement si le thème de la loi applicable aux contrats de consommation doit être régulée dans le système interaméricain par le moyen d'une Convention (tel que le suggère la proposition brésilienne) ou par loi-modèle (comme le suggère la proposition canadienne), et sur celui de la juridiction, par Convention (comme le suggère la proposition uruguayenne de bases pour une Convention en la matière) ou par loi-modèle (comme le suggère la proposition canadienne), ainsi que les autres thèmes de réparation (redress) par loi-modèle (comme le suggèrent les différentès lois-modèles étasuniennes).

La réponse est simple : «le diable se cache dans les détails», comme l'affirme le dicton populaire. Une décision prise avant la réunion, dans l'abstrait et le général, avant l'examen par les experts des propositions et des discussions, aurait été précipitée. La difficulté d'adopter un agenda fructueux pour la CIDIP VII sur la protection des consommateurs ne concerne pas la décision sur un aspect abstrait, mais plutôt sur son texte concret.

Je m'explique. Une excellente idée de loi-modèle conceptuelle et générale, lorsque transformée en différents petits textes, en viendra à présenter plusieurs règles polémiques pour un système juridique et son origine dans la préoccupation pour la protection des consommateurs deviendra alors hésitante, n'atteindra jamais plus un consensus. Il en est de même lorsqu'une loi-modèle sur la juridiction, en principe flexible et intéressante, laisse clairement comprendre que son acceptation des contrats internationnaux de consommation (là où elle serait utile) voudra dire son acceptation de tous les contrats et dispute de consommation ensuite générés, y compris les nationaux (alors qu'il n'y a pas là, de lacune ou de raison pour changer de modèle législatif puisque les Codes de procédures civiles déjà existants sont efficaces). Alors, la loi-modèle la mieux pensée sera refusée puisqu'elle implique le changement de lois nationales efficaces et très utilisées dans le but d'avancer seulement sur des thèmes internationaux. Et, avec les différences de culture juridique existant dans les Amériques, choisir le meilleur modèle législatif du continent ne représentera jamais une décision facile. Tel que l'affirme le dicton, le diable se cache dans les détails.

Un thème sur lequel repose une certitude sur la possibilité d'atteindre un consensus dans le but d'adopter une Convention, comme celui de la loi applicable aux contrats internationaux de consommation, du moins - comme nous l'avons commenté - entre les pays latino-américains, cache un danger semblable. En effet, si le texte de la convention ne reflète pas la symbolique et les aspirations d'avancer dans la protection des consommateurs avec l'application de la loi qui leur est la plus favorable, et, par exemple, ne limite plus l'autonomie des parties ou encore considère toujours la loi des fournisseurs (loi de leur domicile, de leur résidence ou de la maison-mère des foumisseurs, sans aucune autre exigence) comme étant la loi la plus favorable aux consommateurs, il serait alors meilleur d'abandonner le texte et de laisser la protection des consommateurs dans la région telle qu'elle est, c'est-à- 
dire de laisser les juges appliquer presque systématiquement leur lex fori pour protéger les consommateurs en considérant comme impératives toutes les normes nationales de leurs lois de protection. Comme l'affirme le dicton, le diable se cache dans les détails.

Pour cette raison, s'il y a une grande leçon à tirer de la réunion de Porto Alegre et des négociations du groupe de travail et de rédaction informel qui la suivirent, c'est que la pluralité et le dialogue sont possibles. C'est la sagesse particulière de l'agenda double (loi applicable et procédure civile internationale/instruments d'indemnisation) et ouvert (Convention et/ou Loi-modèle) accepté pour la CIDIP VII sur la protection des consommateurs qui permit un dialogue fructueux, sans censure à la pluralité des préoccupations, nécessités et suggestions des membres de l'OÉA en matière de protection des consommateurs, qu'ils soient de tradition civiliste ou du Common Law, qu'ils soient pays industrialisé ou émergents. L'égalité s'est établie dans. la pluralité des modèles et des suggestions. Le dialogue s'effectue dans les différences.

C'est pourquoi il me semble que, tout comme l'affirma Jean-Michel Arrighi lors de l'ouverture de la réunion de Porto Alegre, l'apparente témérité de cet agenda ouvert peut donner un nouvel élan et porter de nouveaux fruits au système interaméricain et, qui sait, la CIDIP VII pourra peut-être approuver conjointement et de façon complémentaire Loismodèles et une Convention afin de faire avancer la protection des consommateurs dans la région.

Cette belle réunion, l'énorme tolérance et la bonne volonté des honorables délégations ainsi que les discussions intelligentes et fructueuses des experts et des délégués servent à confirmer qu'au moins quelques-unes des propositions présentées sont déjà mûres et suffisantes, qu'on a discuté déjà beaucoup à leur sujet, et qu'on a déjà pu atteindre un consensus minimum afin qu'elles soient déposées afin d'être approuvées lors de la conférence finale des États membres pour une CIDIP VII sur la protection des consommateurs. Il me semble que le terrain est préparé et que cette conférence pourrait (et devrait) maintenant être convoquée. 